\title{
Article \\ Reward boosts reinforcement-based motor learning
}



Pierre Vassiliadis,

Gerard Derosiere, Cecile Dubuc, Aegryan Lete,

Frederic

Crevecoeur, Friedhelm C. Hummel, Julie Duque

pierre.vassiliadis@uclouvain. be

Highlights

Reward improves motor learning beyond performance-based reinforcement feedback

Reward boosts specifically reinforcement-based adjustments in motor commands

These effects persist on the following day, in the absence of reward 


\title{
Article \\ Reward boosts reinforcement-based motor learning
}

\author{
Pierre Vassiliadis, ${ }^{1,2,6, *}$ Gerard Derosiere, ${ }^{1}$ Cecile Dubuc, ${ }^{1}$ Aegryan Lete, ${ }^{1}$ Frederic Crevecoeur, ${ }^{1,3}$ \\ Friedhelm C. Hummel, $2,4,5$ and Julie Duque ${ }^{1}$
}

\begin{abstract}
SUMMARY
Besides relying heavily on sensory and reinforcement feedback, motor skill learning may also depend on the level of motivation experienced during training. Yet, how motivation by reward modulates motor learning remains unclear. In 90 healthy subjects, we investigated the net effect of motivation by reward on motor learning while controlling for the sensory and reinforcement feedback received by the participants. Reward improved motor skill learning beyond performance-based reinforcement feedback. Importantly, the beneficial effect of reward involved a specific potentiation of reinforcement-related adjustments in motor commands, which concerned primarily the most relevant motor component for task success and persisted on the following day in the absence of reward. We propose that the long-lasting effects of motivation on motor learning may entail a form of associative learning resulting from the repetitive pairing of the reinforcement feedback and reward during training, a mechanism that may be exploited in future rehabilitation protocols.
\end{abstract}

\section{INTRODUCTION}

Motor skill learning is the process by which the speed and accuracy of movements improve with practice (Krakauer et al., 2019). A significant amount of research has long since demonstrated that motor learning relies on sensory feedback, which allows reducing movement errors (e.g., Shadmehr et al., 2010; Tseng et al., 2007). More recently, some studies have shown that reinforcement feedback, allowing the adjustment of movements based on knowledge of performance, also plays a role in motor learning (Bernardi et al., 2015; Galea et al., 2015; Mawase et al., 2017; Palminteri et al., 2011; Therrien et al., 2016; Wachter et al., 2009). The contribution of reinforcement feedback to motor learning seems to be particularly important when the quality of the available sensory feedback is low (Cashaback et al., 2017; Izawa and Shadmehr, 2011). These observations suggest that reinforcement feedback may be critical for motor rehabilitation (Quattrocchi et al., 2017; Roemmich and Bastian, 2018), where patients often exhibit sensory impairments in addition to their motor disability (Connell, 2008; Hepworth et al., 2016). However, before clinical translation can occur, significant research is required to characterize the optimal conditions in which sensory and reinforcement feedback can improve motor learning.

One key factor that may influence sensory- and reinforcement-based motor learning is motivation (Lewthwaite and Wulf, 2017). This idea is in line with an ethological perspective: in nature, animals are motivated to learn efficiently motor behaviors that have been repetitively associated with rewarding outcomes, in order to increase the likelihood of reaching these outcomes again in the future (Barron et al., 2010; Yamazaki et al., 2016). Whereas past research on motivation has traditionally focused on the impact of reward on decision-making (Balleine and O'Doherty, 2010; Bush et al., 2002; Dayan and Niv, 2008; Derosiere et al., 2017b; 2017a; Gershman and Daw, 2017; Hare et al., 2011; O’Doherty, 2004; Padoa-Schioppa, 2011; Schultz, 2015; Shima and Tanji, 1998), there has been a recent rise in interest regarding its influence on motor learning (Therrien et al., 2016, Mawase et al., 2017, Uehara et al., 2019; Vassiliadis et al., 2019; Chen et al., 2017; Sporn et al., 2020, Vassiliadis and Derosiere, 2020, Holland et al., 2019).

To tackle this issue, previous studies have investigated motor skill learning with different types of reinforcement and reward. This research showed that the combination of reinforcement (providing knowledge of performance) and reward (providing motivation) can influence motor skill learning (e.g., Abe et al., 2011; Steel et al., 2019, 2016; Wachter et al., 2009; Wilkinson et al., 2015). A key aspect of the aforementioned studies is that they considered reinforcement and reward in a bonded way, with the rewarded participants

\footnotetext{
${ }^{1}$ Institute of Neuroscience, Université Catholique de Louvain, 53, Avenue Mounier, Brussels 1200, Belgium

2Defitech Chair for Clinical Neuroengineering, Center for Neuroprosthetics (CNP) and Brain Mind Institute (BMI), Swiss Federal Institute of Technology (EPFL), Geneva 1202, Switzerland

Institute of Information and Communication Technologies, Electronics and Applied Mathematics, Université Catholique de Louvain, Louvain-la-Neuve 1348, Belgium

${ }^{4}$ Defitech Chair for Clinical Neuroengineering, Center for Neuroprosthetics (CNP) and Brain Mind Institute (BMI), Swiss Federal Institute of Technology Sion (EPFL), Sion 1951, Switzerland

${ }^{5}$ Clinical Neuroscience, University of Geneva Medical School (HUG), Geneva 1202 Switzerland

ŁLead contact *Correspondence: pierre.vassiliadis@uclouvain. be

https://doi.org/10.1016/j.isci. 2021.102821
} 
being also the ones receiving performance-based reinforcement feedback. The assumption underlying this approach is that receiving knowledge of performance (e.g., points or binary feedback) provides a form of intrinsic reward that by itself increases motivation to perform well (Leow et al., 2018). However, in addition to the intrinsically rewarding properties of reinforcement, knowledge of performance also provides a learning signal to the motor system that can influence motor learning (Bernardi et al., 2015; Galea et al., 2015; Huang et al., 2011; Kim et al., 2019; Leow et al., 2018; Mawase et al., 2017; Nikooyan et al., 2015; Shmuelof et al., 2012; Therrien et al., 2016; Uehara et al., 2018). In contrast, extrinsic reward increases motivation to perform well without conveying any additional learning signal (Berke, 2018). In accordance with a dissociable role of reinforcement and reward in motor learning, past research has shown that certain subpopulations of neurons in the motor cortex (i.e., a key region of the motor learning network; Krakauer et al., 2019) are responsive to the outcome of previous movements irrespective of reward (Levy et al., 2020), while others respond to reward regardless of the previous outcome (Ramkumar et al., 2016). Put together, these elements indicate that estimating the net impact of reward on motor learning requires controlling for the effect of the reinforcement feedback on the learning process. Based on these elements, we experimentally uncoupled knowledge of performance from reward to test the hypothesis that reward induces a specific improvement in motor skill learning and maintenance.

Another important question relates to how, at the single-trial level, motivation by reward may affect motor skill learning and maintenance. As such, computational models of motor learning posit that movement errors can be corrected based on sensory and reinforcement feedbacks on a trial-by-trial basis (Cashaback et al., 2017), with possible interactions between these two processes (Izawa and Shadmehr, 2011). Sensory-based motor learning relies on the ability to produce motor commands that match predicted sensory consequences (e.g., visual, somatosensory consequences; Sidarta et al., 2016; Bernardi et al., 2015). Conversely, reinforcement-based motor learning is thought to depend on the ability to efficiently regulate between-trial motor variability based on previous outcomes (Dhawale and Smith, 2017; Pekny et al., 2015; Sidarta et al., 2016; Therrien et al., 2016; Uehara et al., 2019; Wu et al., 2014). Importantly, in this framework, reward may have a global influence, enhancing both sensory- and reinforcement-based adjustments from one trial to another, or could have a more specific effect, boosting only one of the two learning systems (Galea et al., 2015; Kim et al., 2019). Here, we investigated the impact of reward on sensory- and reinforcement-based motor adjustments during motor skill learning at the single-trial level, in a situation where they can both contribute to the learning process.

Healthy participants $(n=90)$ trained on a pinch-grip force reproduction task with limited sensory feedback over two consecutive days while we manipulated the reinforcement feedback and reward on Day 1. By removing visual feedback on most trials, we ensured that the learning process would largely depend on the integration of somatosensory and reinforcement feedbacks (Bernardi et al., 2015; Izawa and Shadmehr, 2011; Sidarta et al., 2019). Moreover, subjects were distributed in three groups where training involved sensory (S) feedback only (Group.s; $n=30$ ), sensory and reinforcement (SR) feedback (Group. SR; $\mathrm{n}=30$ ), or both feedbacks and a reward (SRR, Group_sRR; $\mathrm{n}=30$ ). Monetary gains were used as they are known to strongly modulate the motivation to engage in various tasks (Grogan et al., 2020a, 2020b; Manohar et al., 2015; Schultz, 2015; Shadmehr et al., 2019). We investigated how participants learned and maintained the skill depending on the type of feedback experienced during training. We found that while sensory and reinforcement feedbacks were not sufficient for the participants to learn the task in the present study, adding reward during training markedly improved motor performance. Reward-related gains in motor learning were maintained on Day 2, even if subjects were no longer receiving a reward on that day. Importantly, single-trial analyses showed that reward specifically increased reinforcement-related adjustments in motor commands, with this effect being maintained on Day 2, in the absence of reward. The pinch-grip force task used here also allowed considering adjustments separately for the speed of force initiation and the accuracy of the performed force, both in terms of variability and amplitude. Importantly, we found that reward did not affect the control of all motor components in the same way, with the amplitude component turning out to be the more strongly influenced by the presence of reward.

Altogether, the present results provide evidence that motivation by reward can improve motor skill learning and maintenance even when the task is performed with the same knowledge of performance. More importantly, this effect seems to entail a specific potentiation of reinforcement-related adjustments in the motor command at the single-trial level. These behavioral results are important to characterize the 
A

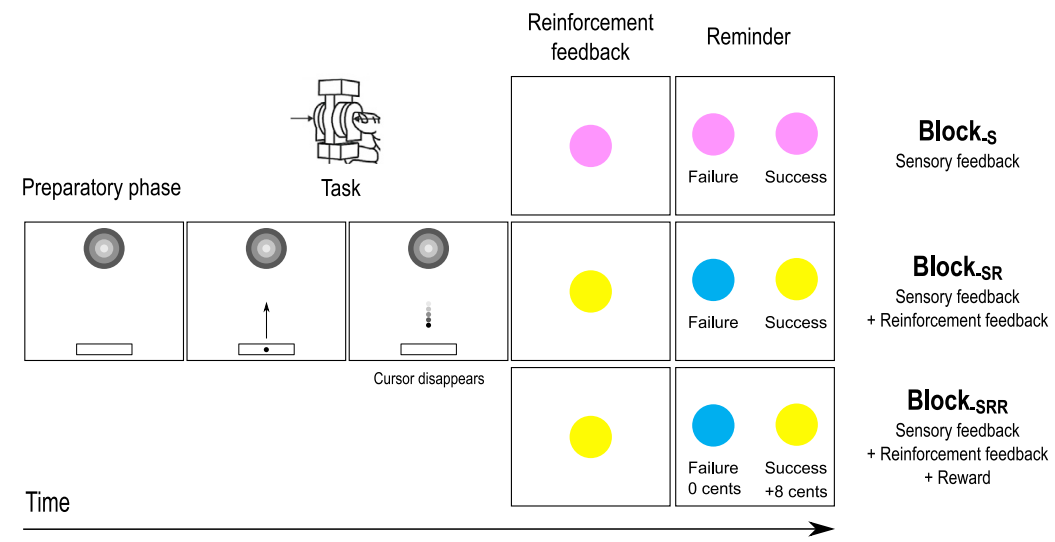

B

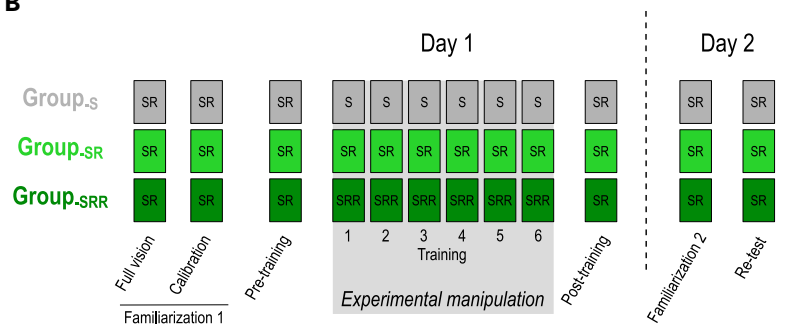

C

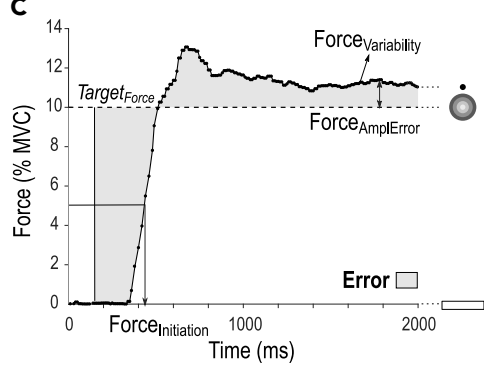

Figure 1. The motor skill learning task

(A) Time course of a trial in the motor skill learning task. Each trial started with the appearance of a sidebar and a target. After a variable preparatory phase $(800-1000 \mathrm{~ms})$, a cursor appeared in the sidebar, playing the role of a "Go" signal. At this moment, participants were required to pinch the force transducer to bring the cursor into the target as quickly as possible and maintain it there until the end of the task $(2000 \mathrm{~ms})$. Notably, on most trials, the cursor disappeared halfway toward the target (as displayed here). Then, a reinforcement feedback was provided in the form of a colored circle for $1000 \mathrm{~ms}$ and provided binary knowledge of performance (Success or Failure in Block-sR and Block_-sRR) or was noninformative (Block-S). The reinforcement feedback was determined based on the comparison between the Error on the trial and the individual success threshold (computed in the Calibration block, see STAR Methods). Finally, each trial ended with a reminder of the color/feedback association and potential reward associated to good performance (1500ms).

(B) Experimental procedure. On Day 1, all participants performed two familiarization blocks in a Block.sR condition. The first one involved full vision of the cursor while the second one provided only partial vision and served to calibrate the difficulty of the task on an individual basis (See STAR Methods). Then, Pre- and Post-training Block_sR assessments were separated by 6 blocks of training in the condition corresponding to each individual group (Block_s for Group_s, Block_sR for

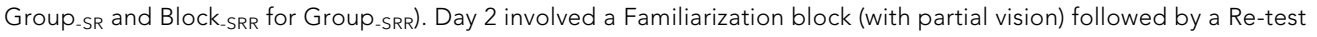
assessment (4 Block_sR pooled together). There was no recalibration on Day 2.

(C) Example of a force profile. Force applied (in \% of MVC) during the task. Participants were asked to approximate the Target $_{\text {Force }}$ as quickly and accurately as possible to minimize the Error (gray shaded area). As shown on the Figure, this Error depended on the speed of force initiation (Force Initiation) and on the accuracy of the maintained force, as reflected by

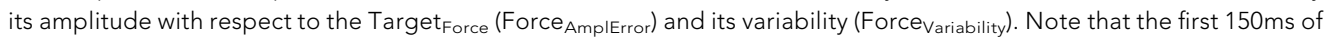
each trial were not considered for the computation of the Error.

mechanisms by which reward can improve motor learning and may guide future motivational interventions for rehabilitation (McGrane et al., 2015).

\section{RESULTS}

Ninety healthy participants practiced a pinch-grip force task over two consecutive days. The task required participants to hold a pinch grip sensor in their right hand and to squeeze it as quickly as possible in order to move a cursor displayed on a computer screen in front of them, from an initial position to a fixed target (Figure $1 \mathrm{~A}$ ). The force required to reach the target (Target Force $_{\text {) }}$ corresponded to $10 \%$ of the individual maximum voluntary contraction (MVC). In most of the trials (90\%), participants practiced the task with very limited sensory feedback: The cursor disappeared when the generated force reached half of the

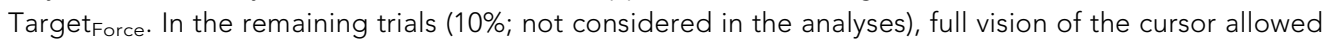




\begin{tabular}{|c|c|c|c|c|c|}
\hline & Group.s $_{(n=30)}$ & Group.SR $(n=30)$ & $\operatorname{Group}_{\text {-SRR }}(\mathrm{n}=30)$ & $\mathrm{F}_{(2,87)}$ & $\mathrm{p}$ \\
\hline Age (years) & $23.9 \pm 0.67$ & $23.3 \pm 0.50$ & $23.9 \pm 0.43$ & 0.34 & 0.71 \\
\hline Gender (number of females) & 19 & 19 & 20 & / & / \\
\hline Success threshold (\% MVC) & $2.8 \pm 0.01$ & $2.8 \pm 0.01$ & $2.9 \pm 0.01$ & 0.13 & 0.88 \\
\hline Target $_{\text {Force }}$ (Newtons) & $5.3 \pm 0.30$ & $4.7 \pm 0.25$ & $5.1 \pm 0.21$ & 1.16 & 0.31 \\
\hline Sensitivity to reward (score) & $37.1 \pm 1.18$ & $35.6 \pm 1.11$ & $37.5 \pm 1.14$ & 0.79 & 0.46 \\
\hline $\begin{array}{l}\text { Sensitivity to punishment } \\
\text { (score) }\end{array}$ & $42.3 \pm 1.59$ & $42.0 \pm 1.59$ & $40.9 \pm 1.46$ & 0.22 & 0.81 \\
\hline $\begin{array}{l}\text { Muscle fatigue - Day } 1 \\
\left(\text { MVC }_{\text {POST }} \text { in \% of MVC } C_{\text {PRE }}\right)\end{array}$ & $95.7 \pm 2.39$ & $99.0 \pm 2.61$ & $98.6 \pm 2.57$ & 0.51 & 0.60 \\
\hline $\begin{array}{l}\text { Muscle fatigue - Day } 2 \\
\left(\mathrm{MVC}_{\mathrm{POST}} \text { in \% of } \mathrm{MVC} \mathrm{C}_{\mathrm{PRE}}\right)\end{array}$ & $94.6 \pm 2.74$ & $93.9 \pm 1.52$ & $97.0 \pm 1.89$ & 0.60 & 0.55 \\
\hline
\end{tabular}

participants to be visually guided toward the Target Force $_{\text {and }}$ therefore to be reminded of the somatosensory sensation corresponding to the Target Force. Hence, in this task, learning relied mostly on the successful

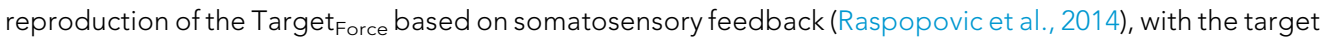
somatosensory sensation being regularly reminded to the participants through the full vision trials. To learn the task, subjects were provided with six training blocks ( 40 trials each; i.e., total of 240 training trials; Figure 1B), during which Group_s subjects trained with sensory feedback only (Block_s), Group_sR subjects trained with sensory and reinforcement feedback (Block_sR), and Group_sRR subjects trained with both feedbacks and a monetary reward (Block_sRR). Notably, the groups were comparable for a variety of features

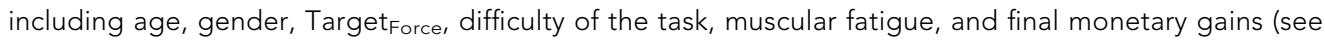
STAR Methods, Table 1). Beside the training blocks, all participants performed the task in a Block_sR setting so that the familiarization, the pre- and post-training assessments on Day 1, as well as Re-test on Day 2, occurred in the same conditions in the three experimental groups. This design allowed us to investigate the effect of reinforcement and reward, both on learning and on maintenance of the learned motor skill. Importantly, in Block_sR and Block_sRR, the binary reinforcement feedback depended on the Error, estimated as the absolute difference between the Target $_{\text {Force }}$ and the exerted force over the whole trial (excluding the first 150 ms, Figure 1C; Abe et al., 2011; Steel et al., 2016). Hence, to be successful, partic-

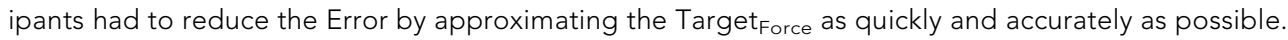

\section{Reward improves motor skill learning}

Participants' initial performance was comparable in all groups: The Error in the Pre-training block equaled $3.14 \pm$ $0.18 \%$ MVC in Group_s, $3.33 \pm 0.17 \%$ MVC in Group_sR and $3.30 \pm 0.15 \%$ MVC in Group_sRR (one-way ANOVA: $F_{(2,87)}=0.37, p=0.69$, partial $\eta^{2}=0.0084 ;$ Figure $\left.2 A\right)$. In contrast, skill learning, estimated as the training-related reduction in Error on Day 1 (Normalized Error = Post-training Error expressed in \% of Pre-training Error) varied as a function of the group (Figure 2B). As such, learning was stronger in the Group-sRR compared to the two other groups (ANOVA: $F_{(2,87)}=4.41, p=0.015$, partial $\eta^{2}=0.092$; post-hocs: Group_sRR Vs. Group_sR: $p=0.014$, Cohen's $d=0.60$; Group_sRR Vs. Group $s$ : $p=0.010, d=0.98$, with no significant difference between Group_s and Group -SR $_{\text {-S }}$ $(p=0.91, d=0.025)$. This was confirmed by a subsequent analysis showing that learning was significant in the Group-SRR (Post-training $=80.7 \pm 3.5 \%$ of Pre-training; single-sample ttest against $100 \%: \mathrm{t}_{(29)}=-5.49, \mathrm{p}<$ $0.00001, d=-1.42$ ), but not in Group - (Post-training $=103.9 \pm 5.15 \%$ of Pre-training; $t_{(29)}=0.75, p=0.46$, $d=0.19$ ) or in Group-SR (Post-training $=102.9 \pm 8.80 \%$ of Pre-training; $\left.t_{(29)}=0.33, p=0.745, d=0.085\right)$. Skill maintenance on Day 2, estimated as the Error at Re-test in percentage of Pre-training, was not significantly different between the groups $\left(F_{(2,87)}=1.96, p=0.15\right.$, partial $\eta^{2}=0.043$; Figure $\left.2 C\right)$. However, in Group-SRR, we found that the Error at Re-test remained lower than at Pre-training (Re-test $=85.6 \pm 5.01 \%$ of Pre-training; single-sample ttest against $100 \%$ : $\left.\mathrm{t}_{(29)}=-2.88, \mathrm{p}<0.0073, \mathrm{~d}=-0.74\right)$ demonstrating that the skill was maintained, while this effect was not significant in the two other groups (Group_s: Re-test $=100.5 \pm 4.63 \%$ of Pretraining; $\mathrm{t}_{(29)}=0.11, \mathrm{p}=0.92, \mathrm{~d}=-0.12$, Group_sR: Re-test $=97.0 \pm 6.82 \%$ of Pre-training; $\mathrm{t}_{(29)}=-0.45, \mathrm{p}=$ $0.66, d=-0.028)$. Hence, while reinforcement alone did not contribute to reduce the Error in this task, its combination with reward successfully helped participants to learn and maintain the skill, as also evident when considering the averaged success rates (Figure 2D) and individual force profiles (Figure 2E). 
A

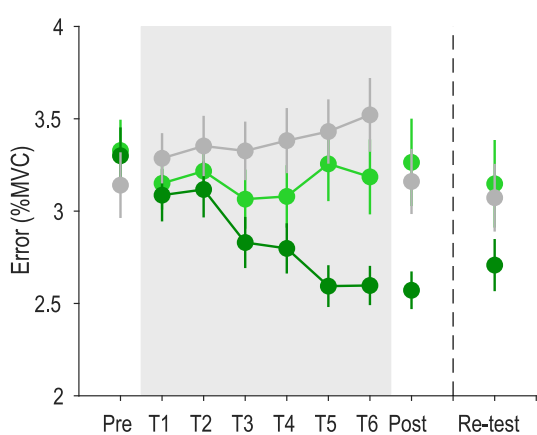

B

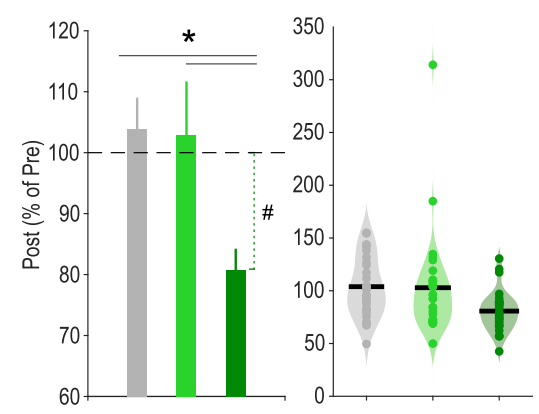

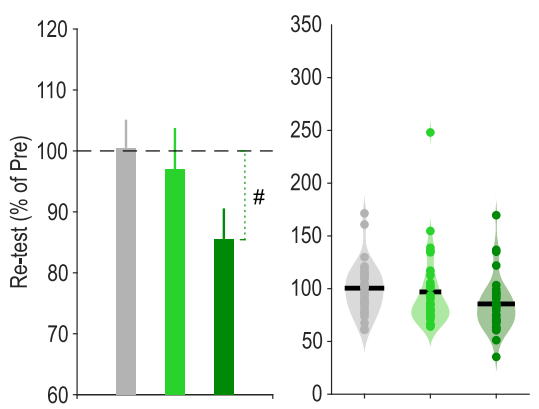

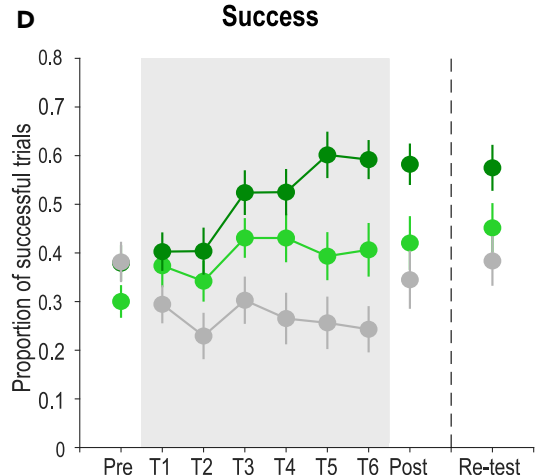

E
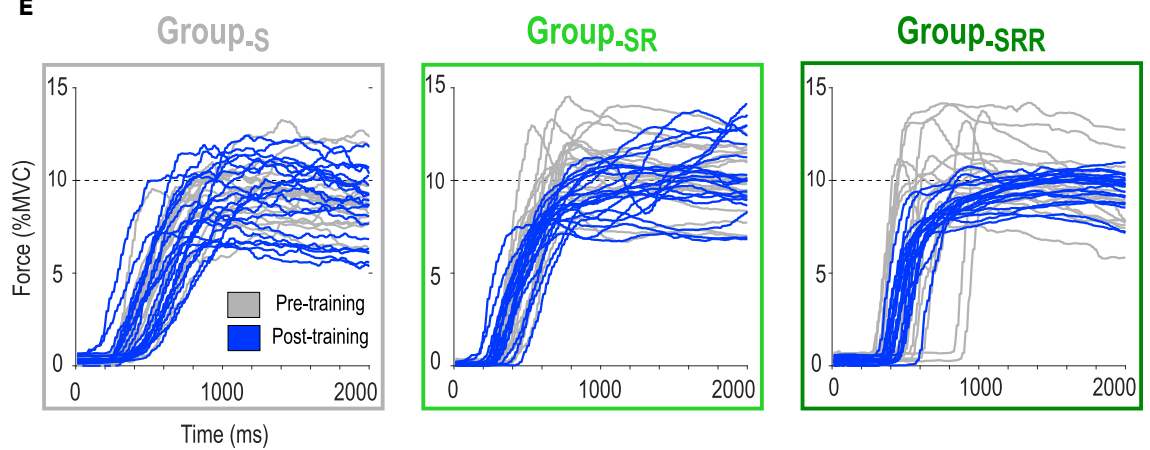

Figure 2. Effect of reward on motor skill learning

(A) Error. Average Error is represented across practice for the three experimental groups (gray: Group_s, light green: Group_sR, dark green: Group_sRR). The gray shaded area highlights the blocks concerned by the reinforcement manipulation. The remaining blocks were performed with knowledge of performance only (i.e., in a Block-sR setting).

(B) Skill learning. Bar plot (left) and violin plot (right, each dot = one subject) representing skill learning (quantified as the Error in Post-training blocks expressed in percentage of Pre-training blocks) in the three experimental groups. Skill learning was significantly enhanced in Group-sRR compared to the two other groups. This result remained significant when removing the subject showing an extreme value in the Group_sR (ANOVA: $F_{(2,86)}=6.44, p=0.0025, p a r t i a l$ $\eta^{2}=0.13$; post-hocs; Group-SRR vs. Group-SR: $p=0.027$; Group-SRR vs. Group-S: $p=0.00064$; Group-SR vs. Group-S: $\left.p=0.21\right)$.

(C) Skill maintenance. Bar plot (left) and violin plot (right) representing skill maintenance quantified as the Error in Re-test blocks expressed in percentage of Pre-training blocks) in the three experimental groups.

(D) Success. Proportion of successful trials for each block.

(E) Force profiles. Individual force profiles of one representative subject of Group_s (left), Group_sR (middle) and Group_sRR (right) in the Pre- (gray) and Posttraining blocks (blue). Note the better approximation of the Target Force $_{\text {and }}$ the reduced inter-trial variability at Post-training in the exemplar subject of

Group-SRR.

*: significant difference between groups $(p<0.05)$. \#: significant difference within a group between normalized Post-training Error and a constant value of $100 \%$ ( $p<0.017$ to account for multiple comparisons). Data are represented as mean $\pm \mathrm{SE}$

\section{Reward boosts reinforcement-related adjustments during motor skill learning}

To identify the mechanisms at the basis of the effect of reward on motor learning, we quantified how much participants adjusted motor commands based on reinforcement or sensory feedback the single-trial level. This allowed us to estimate how subjects relied on each type of feedback on a trial-by-trial basis and how this behavior was affected by reward. In order to investigate reinforcement-related adjustments in motor commands,

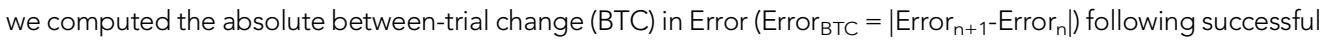
or failed trial ${ }_{n}$ of similar Error in the three groups (STAR Methods, see also (Pekny et al., 2015; Uehara et al., 2019) for similar approaches in reaching tasks). Comparing Error BTC depending on the Outcome of the previous trial (Success or Failure) allowed us to estimate how much participants modified their force profile based on the reinforcement feedback. Notably, considering changes in the Error in absolute terms allowed us to explore the effect of reward on the magnitude of the adjustments in the different groups, regardless of their directionality (increase or decrease in the Error). We found that Error ${ }_{\text {BTC }}$ was generally higher after failed than successful trials (two-way ANOVA; main effect of Outcome: $F_{(1,84)}=8.66, p=0.0042$, partial $\eta^{2}=0.093$; Figure $3 A$ ), consistent with an exploration process following failed trials (Uehara et al., 2019; Pekny et al., 2015). Interestingly, this difference 


\section{Reinforcement-based adjustments in motor commands}
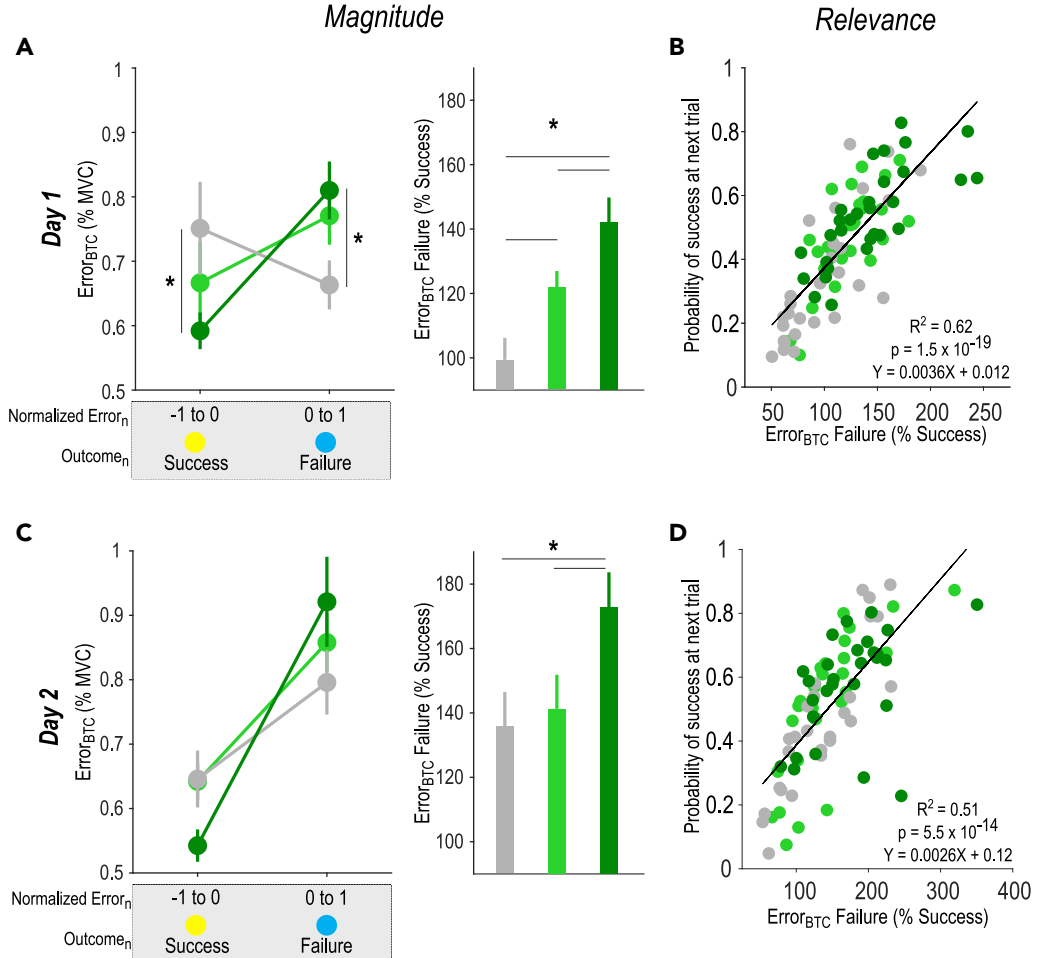

Sensory-based adjustments in motor commands
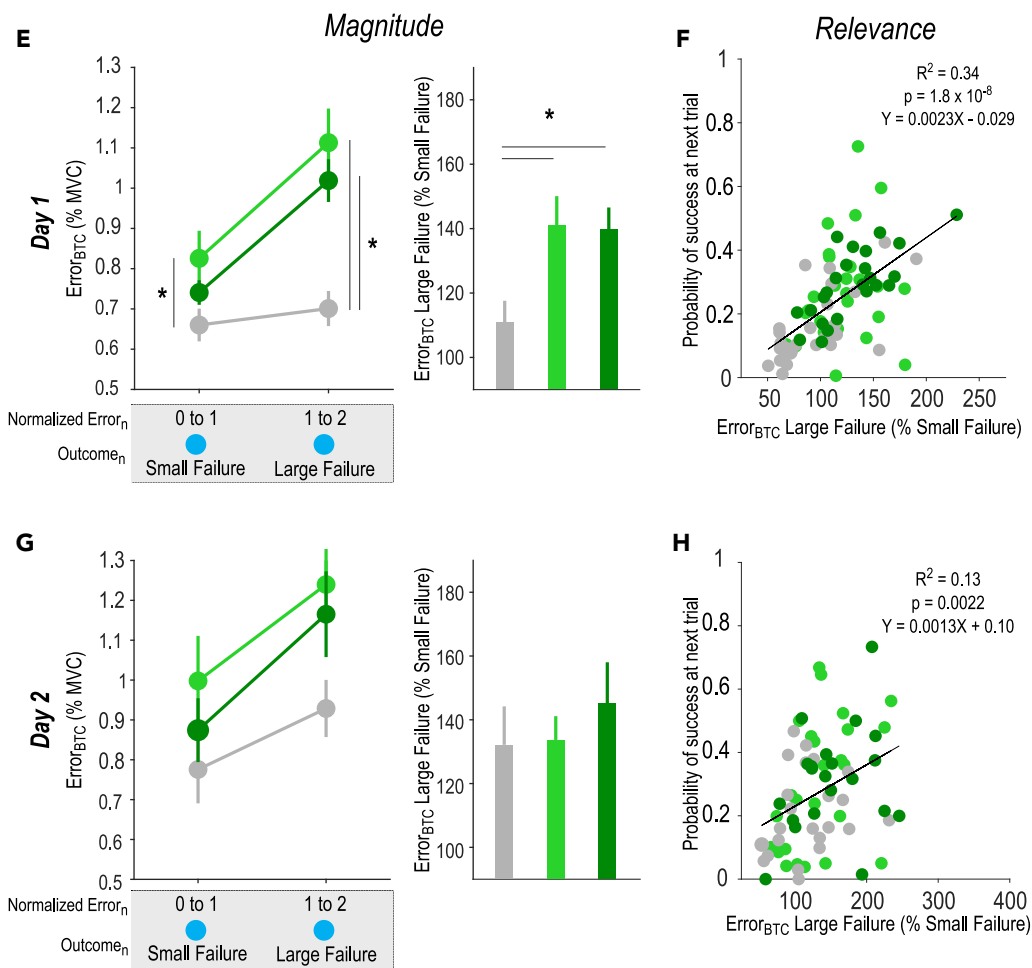

Figure 3. Between-trial adjustments in the Error

(A) Reinforcement-based adjustments in the Error during Day 1 training. Absolute between-trial adjustments in the Error $\left(\right.$ Error $_{\mathrm{BTC}}=\mid$ Error $_{\mathrm{n}+1}-$ Error $\left._{\mathrm{n}} \mid\right)$ according to the reinforcement feedback (i.e., Success or Failure) encountered at trial $\mathrm{n}_{\mathrm{n}}$ in the 
Figure 3. Continued

three GrouptYPES (gray: Group_s, light green: Group_sR, dark green: Group_sRR). Notably, these bins of trials were constituted based on the success threshold-normalized Error at trial $\mathrm{n}_{\mathrm{n}}$ in order to compare adjustments in motor commands following trials of similar Error in the three groups. Stars denote significant group differences in Error ${ }_{\text {BTC }}$ for a given outcome (left panel, see STAR Methods). Reinforcement-based adjustments (Error $r_{B T C}$ after Failure in percentage of Error BTC after Success) were compared in the three GroupTYPES (right panel).

(B) Correlations between the magnitude of reinforcement-based adjustments in the Error and the average success rate on the next trial, showing the relevance of these adjustments in the present task. Each dot represents a subject.

(C, D) Same for Day 2 training. Note that reinforcement-based adjustments in motor commands remained amplified in GroupsRR, despite the absence of reward on Day 2.

(E) Sensory-based adjustments in the Error during Day 1 training. Error ${ }_{\mathrm{BTC}}$ following trials $\mathrm{s}_{\mathrm{n}}$ with Failures of different Error magnitudes (left panel). Sensory-based adjustments (Error BTC after Large Failure in percentage of Error ${ }_{\text {BTC }}$ after Small Failure) were compared in the three Group TYPES (right panel).

(F) Correlations between the magnitude of sensory-based adjustments in the Error and the probability of success on the next trial, showing the relevance of these adjustments for task success.

$(G, H)$ Same for Day 2 training. *: $p<0.05$. Data are represented as mean \pm SE.

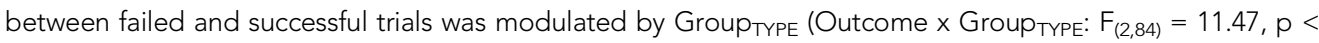
0.001, partial $\eta^{2}=0.21$ ): While it was significant in Group_sR and Group_sRR (post-Success vs. post-Failure: $p=$ $0.028, d=-0.54$ and $p<0.001, d=-0.92$ respectively), this effect was only at the trend level for Group-s $(p=0.060, d=0.29)$. Relatedly, post-hoc tests revealed that post-Success Error BTC was significantly lower in Group_sRR $_{\text {-SRan in Group_S }}(p=0.026, d=-0.54)$, but not different between Group_sR and Group_S $(p=0.24$,

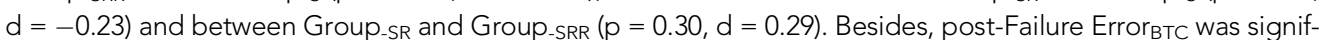
icantly higher in Group_SRR than in Group_s $(p=0.040, d=0.65)$. Yet, it was not different between Group_sR and $\operatorname{Group}_{\text {SS }}(p=0.14, d=0.48)$ and between Group_SR and Group_SRR $(p=0.58, d=-0.16)$. Hence, providing a reward on top of reinforcement feedback led to a particularly low Error ${ }_{B T C}$ following successful trials and a partic-

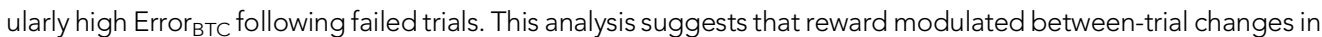
behavior in response to the reinforcement feedback, regardless of whether reinforcement was positive or negative. To further confirm this, we directly compared the magnitude of reinforcement-based adjustments between the three groups, by expressing the Error ${ }_{\mathrm{BTC}}$ following failed trials relative to the Error ${ }_{\mathrm{BTC}}$ following successful trials. Doing so, we found a significant effect of the GroupTYPE during Day 1 training $\left(F_{(2,84)}=10.27, p<0.001\right.$, partial $\eta^{2}=0.20$; Figure 3A). As expected, participants of the Group-sR adjusted their force profile depending on the reinforcement feedback, while participants of the Groupss were unable to do so (post-hocs; Group_s vs. Group_sR: $p=0.022, d=-0.68$ ). Interestingly, this ability to adjust motor commands based on the reinforcement was amplified by reward (Group_SR Vs. Group_sRR: $p=0.036, d=-0.57$ ). This result suggests that one mechanism through which reward improves motor learning is the potentiation of reinforcement-related adjustments in motor commands. To further test this idea, we evaluated the relationship between the magnitude of reinforcement-based changes in motor commands and the average success rate in the following trial across all subjects. Consistently, we found that the magnitude of reinforcement-related adjustments was strongly associated to the probability of success $\left(R^{2}=0.62 ; p=1.5 \times 10^{-19}\right.$; Figure $\left.3 B\right)$ : The more participants adjusted their behavior based

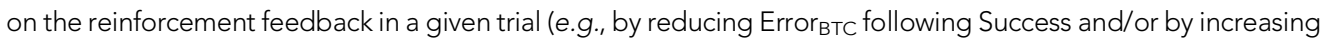
it following a Failure), the more they were likely to be successful in the following trial, supporting the view that these adjustments were relevant in the present task. Hence, these data suggest that the effect of reward on motor skill learning relies on the ability to adjust movements based on the reinforcement feedback.

In the second step, we asked whether such single-trial effects were maintained on Day 2, while all participants performed the task with sensory and reinforcement feedback, but in the absence of reward (i.e.,

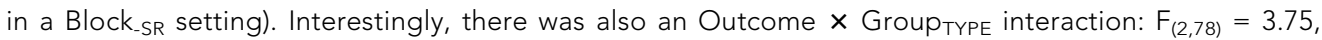
$p=0.027$, partial $\eta^{2}=0.088$ ) demonstrating differences in the way participants relied on the reinforcement feedback on Day 2 based on the type of training experienced on Day 1 (Figure 3C). All groups displayed a

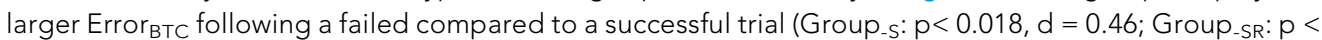
$0.001, d=0.75$; Group_sRR: $p<0.001, d=1.13$ ). Notably though, post-hoc tests did not identify any group difference in post-Success Error BTC $_{\text {BT }}$ (Group_sR vs. Group_sRR: $p=0.13, d=0.72$; Group_sR vs. Group_s: $p=$ $0.96, d=-0.019 ;$ Group_sRR vs. Group_s: $p=0.12, d=-0.56$ ) nor did it do so in post-Failure Error BTC (Group. SR vs. Group_sRR: $p=0.33, d=-0.20$; Group_sR vs. Group_s: $p=0.35, d=0.25$; Group_sRR vs. Group_s: $p=$ $0.058, d=0.39)$. Yet, when expressing Error $r_{\text {BTC }}$ in Post-Failure relative to Post-Success trials, we found that participants receiving reward in Group_SRR adjusted more of their movements according to the reinforcement feedback compared to Group_s and $\operatorname{Group}_{\text {-SR }}\left(F_{(2,78)}=3.53, p=0.034\right.$, partial $\eta^{2}=0.083$; post-hocs; Group_s vs. Group_SRR: $p=0.017, d=-0.66$, Group_sR vs. Group_SRR: $p=0.039, d=-0.56$, Figure $3 C$ ). There 
was no difference between Group_s and Group_sR $(p=0.72, d=-0.10)$. Here, again, the magnitude of reinforcement-based adjustments correlated with the success in the next trial $\left(R^{2}=0.51 ; p=5.5 \times 10^{-14}\right.$; Figure 3D). Hence, the effect of reward on reinforcement-based adjustments can persist on a subsequent session of training, even after reward removal.

As explained above, we evaluated reinforcement-based adjustments by comparing Error BTC $_{\text {following suc- }}$ cessful or failed trials. However, by definition, successful and failed trials did not only differ with respect to the reinforcement feedback obtained at trial $\mathrm{l}_{n}$, but also regarding the experienced sensory feedback. Hence, the reward effect reported above could be specific to reinforcement-based adjustments, or may reflect a different reliance on the sensory feedback (or a combination of both). To disentangle these possibilities, we reasoned that the extent to which participants relied on the somatosensory feedback to adjust their movements could be estimated by computing Error ${ }_{\mathrm{BTC}}$ following failed trials of different Error magnitudes (i.e., small or large Failure). In other words, we contrasted Error ${ }_{\mathrm{BTC}}$ following trials with the same reinforcement feedback (i.e., Failure) but with different somatosensory experiences (i.e., resulting from Small or Large Failures). Here too, we found a significant Outcome $\times$ Group TYPE interaction on the Error $_{\text {BTC }}$ $\left(F_{(2,76)}=5.15, p=0.0080\right.$, partial $\eta^{2}=0.12$; Figure $\left.3 E\right)$. As such, adjustments were greater after Large than after Small Failures in Group_SR and Group_SRR $(p<0.001, d=0.65$ and $p<0.001, d=1.20$, respectively), but not in Group.s $(p=0.50, d=0.19)$. Post-hoc tests also indicated that adjustments after a Large Failure were

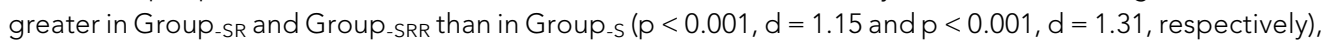
but not different between Group_SR and Group-SRR $(p=0.26 p<0.001, d=0.25)$. After Small Failures, Error $_{\text {BTC }}$ was also larger in Group.SR than in Group.s $(p=0.042, d=0.56$ ), but not different between Group_SR $_{\text {and Group_SRR }}(p=0.31, d=0.30)$ and between Group_SRR and Group_s $(p=0.34, d=0.44)$. This indicates that while subjects of the Group_sR and Group_-SRR adjusted the Error depending on the sensory feedback, participants of the Group.s were not able to do so, suggesting that training with reinforcement feedback allowed participants to be more sensitive to the sensory feedback (Galea et al., 2015; Bernardi et al., 2015), regardless of whether they received reward or not. Consistently, we found a Group TYPE effect $\left(F_{(2,76)}=5.05, p=0.0087\right.$, partial $\eta^{2}=0.12$; Figure $\left.3 E\right)$ on the magnitude of sensory-based adjust-

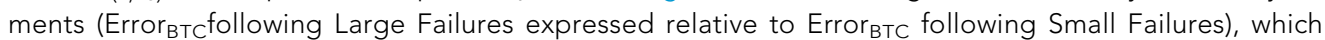
was driven by differences between Group_s and the two other groups (post-hocs; Group.s vs. Group-sR: $p=0.0056, d=-0.73$, Group_s vs. Group_sRR: $p=0.011, d=-0.85)$. Importantly, we did not find any difference between Group-SR and Group-SRR ( $p=0.90, d=-0.033$ ). Then, similarly as for the reinforcement-based changes, we found that the magnitude of sensory-based adjustments correlated with the subsequent probability of success $\left(R^{2}=0.34, p=1.8 \times 10^{8}\right.$; Figure $\left.3 F\right)$, demonstrating that these adjustments were also relevant in the learning process.

On Day 2, the effect of Outcome persisted $\left(F_{(1,68)}=15.20, p<0.001\right.$, partial $\left.\eta^{2}=0.18\right)$ with a trend for a Group TYPE $_{\text {effect }}\left(F_{(2,68)}=3.12, p=0.051\right.$, partial $\left.\eta^{2}=0.084\right)$ but no Outcome $\times$ Group TYPE interaction $\left(F_{(2,68)}=3.12, p=0.46\right.$, partial $\left.\eta^{2}=0.013\right)$. Consistently, the magnitude of sensory-based adjustments was not different between the Group TYPES $\left(F_{(2,68)}=0.41, p=0.67\right.$, partial $\eta^{2}=0.012$, Figure $\left.3 G\right)$. Note, though, that similarly to Day 1 , sensory-based adjustments significantly correlated with the probability of success on Day $2\left(R^{2}=0.13, p=0.0022\right.$; Figure $\left.3 H\right)$. Hence, the absence of reward effects on sensorybased adjustments on Day 1 and 2 cannot be explained by the fact that participants did not rely on this type of feedback.

The single-trial analyses on Error ${ }_{\text {BTC }}$ revealed significant differences in the way participants of each group adjusted their motor commands based on the reinforcement and the sensory feedback. However, the distribution of the Error data could have contributed to these single-trial effects. Indeed, even for random adjustments in motor commands (e.g., based on a Gaussian process), adjustments following small or large Error $_{n}$ (i.e., in the tails of the Error distribution) would be larger than adjustments following Error ${ }_{n}$ close to the mean of the distribution. Hence, to ensure that group differences in Error distribution did not contribute to our single-trial results, we ran a control analysis in which we shuffled the Error data for each subject (with 10,000 permutations), and then re-computed reinforcement and sensory-based adjustments exactly as in the main analysis. Importantly, we did not find any GroupTYPE effect on these shuffled data neither for reinforcement- (Day 1: $F_{(2,84)}=1.6, p=0.21$, partial $\eta^{2}=0.04$; Day 2: $F_{(2,78)}=0.89, p=0.41$, partial $\eta^{2}=0.02$ ) nor for sensory-based adjustments (Day 1: $F_{(2,76)}=0.02, p=0.98$, partial $\eta^{2}=0.0006$; Day 2: $F_{(2,78)}=0.20, p=0.82$, partial $\eta^{2}=0.006$ ). This analysis indicates that the differences in single-trial adjustments reported here were not related to a sampling bias. 


\section{Reward boosts reinforcement-based adjustments at a specific level of motor control}

As a last step, we asked whether the effect of reward on between-trial adjustments in motor commands concerned all aspects of force control or only some specific motor components. To do so, we investigated how reinforcement and sensory feedback shaped adjustments in the speed and accuracy of force production in the three GroupTYPES by dissecting each force profile into three separate components (Figure 1B). To evaluate the speed at which the force was generated, we computed the time required for force initiation (i.e., the time required to reach half of the Target Force: $_{\text {Force }}$ Initiation). To assess the accuracy of the force, we computed the force difference between the average amplitude of the generated force and the Target Force $_{\text {(Force }}$ AmplError), and the variability (standard deviation/mean) of the maintained force (Force Variability). Notably, both indicators of force accuracy were computed in the second half of the trial (i.e., the last $1000 \mathrm{~ms}$ ), well after force initiation, when participants maintained a stable level of force.

We compared between-trial changes in Force $\left.\right|_{\text {Initiation }}$ (Force Initiation-BTC), Force $_{\text {AmplError }}$ (Force AmplError-BTC), and $_{\text {A }}$ Force Variability $_{\text {(Force }}$ Variability-BTC) following Success or Failure trials of similar Error magnitude in the three groups.


7.62, $p<0.001$, partial $\left.\eta^{2}=0.15\right)$ that was driven by the fact that post-Success and post-Failure Force Initiation-BTC $_{\text {B }}$ were different in Group_SR and Group_SRR $(p<0.001, d=-1.04$ and $p<0.001, d=-1.59$, respectively) but not in $\operatorname{Group}_{-s}(p=0.10, d=-0.27)$. Moreover, post-Success changes in Force Initiation were smaller in Group_sRR than in $\operatorname{Group}_{-s}(p=0.023, d=-0.56)$; it also tended to be smaller in Group_sR than in Group_s $(p=0.071, d=-0.49)$, while it was comparable in Group_sR and Group_-SRR $(p=0.65, d=0.14)$. Corroborating these results, we found that reinforcement feedback impacted the modulation of initiation speed (expressed as Force Initiation-BTC following a Failure in percentage of Force Initiation-BTC following a Success; $F_{(2,84)}=8.50, p<0.001$, partial $\eta^{2}=$ 0.17; post-hocs: Group_s vs. Group_sR: $p=0.0011, d=-0.84$, Group_s vs. Group_sRR: $p<0.001, d=-1.09$; Figure 4A). Interestingly though, we did not find any effect of reward on the reinforcement-based adjustment of

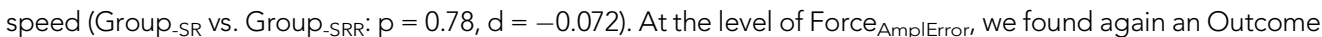
$\times$ Group TYPE interaction $\left(F_{(2,84)}=14.07, p<0.001\right.$, partial $\eta^{2}=0.25$; Figure $\left.4 B\right)$ that was driven by the fact that post-Success and post-Failure Force AmplError-BTC $_{\text {were different in Group_sR and Group_sRR }}(p<0.001, d=$ -0.97 and $p=0.0034, d=-1.44$, respectively) but not in Group -s $(p=0.99, d=-0.0002)$. Group comparisons

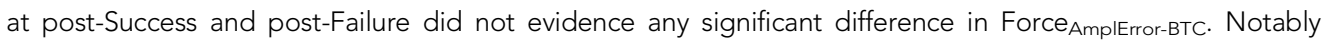
though, there was a trend for the post-Success Force AmplError-BTC to be smaller in Group_sRR than in Group_s ( $p=0.066, d=-0.42)$. Interestingly, direct comparison of reinforcement-related changes in Force AmplError-BTC (post-Failure vs. post-Success) revealed a significant effect of reward $\left(F_{(2,84)}=9.54, p<0.001\right.$, partial $\eta^{2}=$ 0.19; Figure 4B). As such, participants of the Group_sRR modulated more the Force ${ }_{\text {AmplError }}$ according to the reinforcement feedback than subjects of the two other groups (Group_s vs. Group_sRR: $p<0.001, d=-1.04$, Group-sR vs. Group_sRR: $p=0.018, d=-0.70$ ). Notably, there was also a trend for Group_sR to be different from Group_s $(p=0.064, d=-0.50)$. Finally, analysis of Force $V_{V a r-B T C}$ did not reveal any Outcome $\times$ Group TYPE interaction $\left(F_{(2,84)}=0.79, p=0.46\right.$, partial $\eta^{2}=0.018$; Figure $\left.4 C\right)$, neither did it show a Group TYPE effect $\left(F_{(2,84)}=0.81, p=\right.$ 0.45 , partial $\eta^{2}=0.020$; Figure $4 C$ ) on reinforcement-based adjustments (Force var-BTC post-Failure vs. post-Success). Hence, while reward strongly influenced reinforcement-based adjustments of force amplitude, it did not modulate the between-trial regulation of the speed at which the force was initiated or the variability of the maintained force. This suggests that the effect of reward on reinforcement-related adjustments was not global (i.e., affecting all aspects of the movement) but rather specific to force amplitude.

We also considered the effect of the sensory feedback on between-trial adjustments by comparing

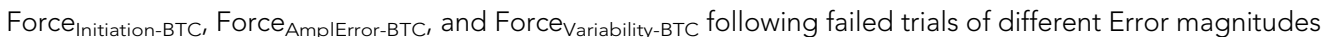
(i.e., small or large Failure). Contrary to the global Error ${ }_{\text {BTC }}$ index, we did not find any Outcome $\times$ Group $_{\text {TYPE }}$ interaction neither for Force Initiation-BTC $\left(F_{(2,76)}=0.54, p=0.59\right.$, partial $\left.\eta^{2}=0.014\right)$, nor for Force AmplError-BTC $_{\text {, }}$ $\left(F_{(2,76)}=2.80, p=0.067\right.$, partial $\left.\eta^{2}=0.069\right)$ or Force Variability-BTC $\left(F_{(2,76)}=1.25, p=0.29\right.$, partial $\left.\eta^{2}=0.032\right)$. Consistently, we did not find any significant difference in the way participants from the different groups adjusted individual motor components depending on the size of the preceding Failure (Large vs. Small Failure on Figures 4D-4F; Force Initiation-BTC: $F_{(2,76)}=0.10, p=0.90$, partial $\eta^{2}=0.0026$; Force AmplError-BTC: $_{(2,76)}=2.57$, $p=0.083$, partial $\eta^{2}=0.063$; Force ${ }_{\text {Variability-BTC: }} F_{(2,76)}=2.46, p=0.092$, partial $\left.\eta^{2}=0.061\right)$. This analysis supports the idea that reward did not increase the sensitivity to the sensory feedback, but rather boosted specific adjustments in motor commands in response to the reinforcement feedback.

Finally, as a control analysis, we characterized the respective influence of each motor component in the Error, which determined task success. As such, in addition to representing different levels of force control 
Reinforcement-based adjustments
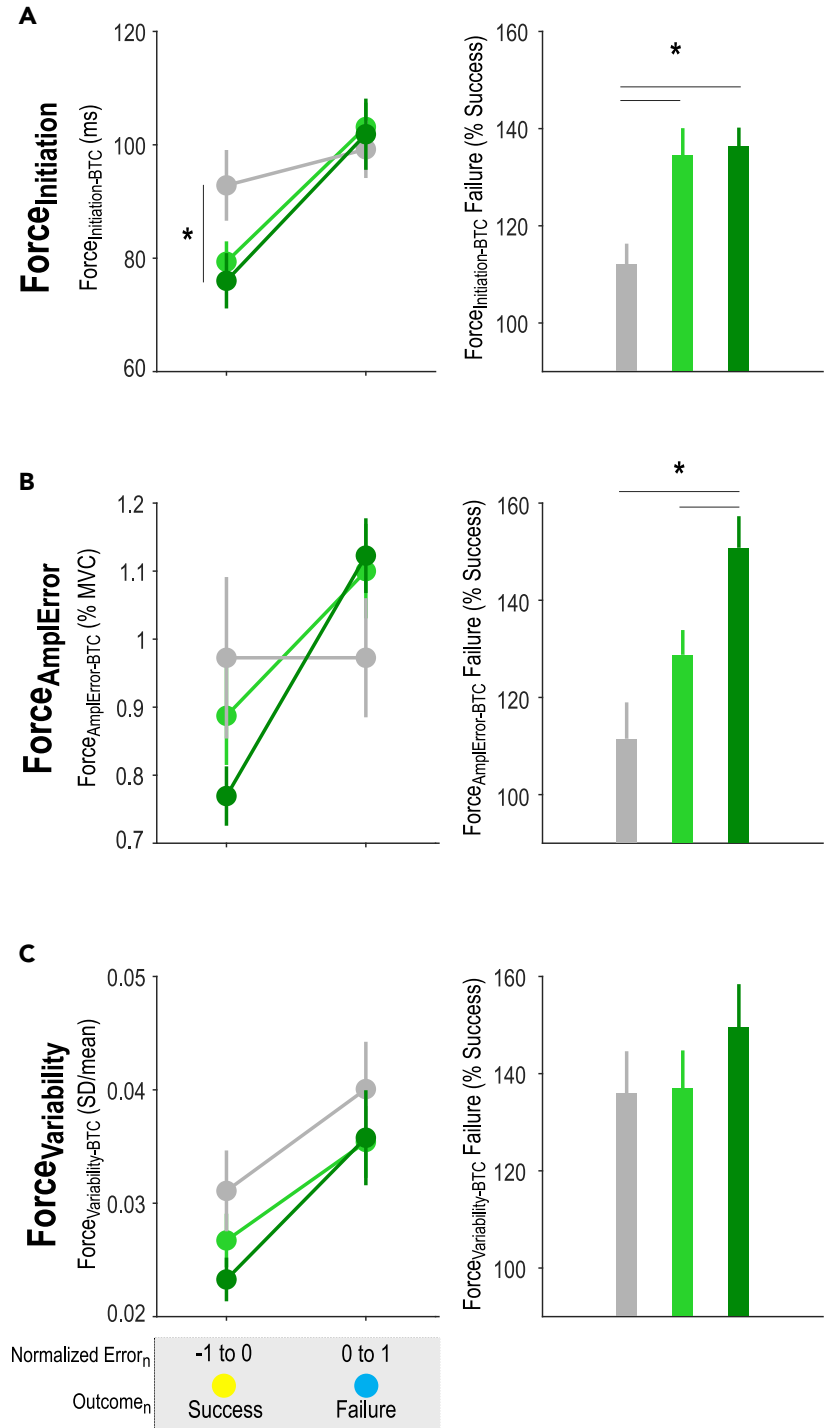

\section{Sensory-based adjustments}


E
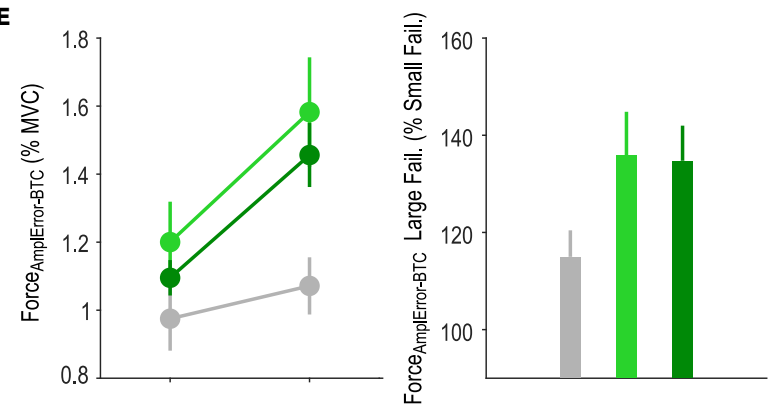

$\mathbf{F}$

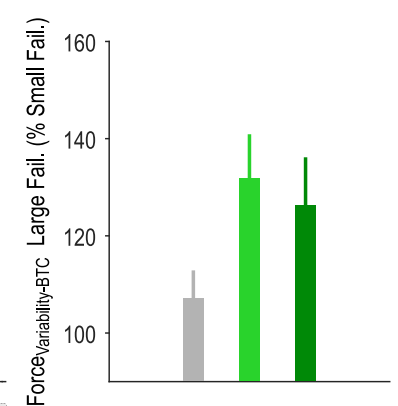

Figure 4. Between-trial adjustments in initiation time, amplitude error and variability

Reinforcement-based adjustments in the Force Initiation (A), Force AmplError $_{\text {(B) and Force }}$ ariability $(C)$. Absolute between-trial changes (BTC) for each motor

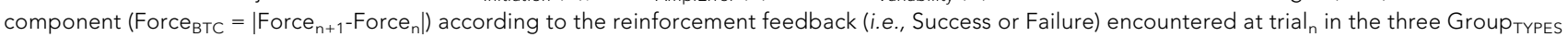
(gray: Group_s, light green: Group_sR, dark green: Group_sRR). Notably, these bins of trials were constituted based on the success threshold-normalized Error


percentage of Force BTC $_{\text {after Success) in the three Group }}$ TYPES (right panel). Sensory-based adjustments in the Force Initiation $_{\text {(D), Force }}$ AmplError $_{\text {(E) and }}$

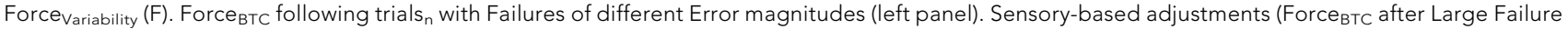
in percentage of Force ${ }_{\mathrm{BTC}}$ after Small Failure) in the three Group TYPES (right panel). *: $\mathrm{p}<0.05$. Data are represented as mean \pm SE.

(i.e., initiation, amplitude and variability), the motor components evaluated here may also bear different relevance for task success (van der Kooij et al., 2021). For each participant, we ran separate partial linear regressions on the Error data with Force Initiation, Force AmplError or Force variability as predictors. Notably, we used partial regressions here to assess the relationship between the Error and each motor component, while controlling for the effect of the other motor components in the correlation. Interestingly, we found that Force AmplError explained the largest part of variance in the Error $(r=0.96 \pm 0.003 ; p<0.05$ in $90 / 90$ sub-

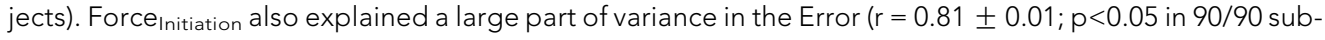

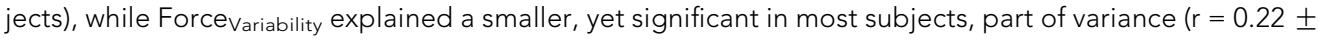


$0.03 ; p<0.05$ in 68/90 subjects). Hence, although all motor parameters were relevant for task success, the Force $_{\text {AmplError }}$ was the most influential factor.

Altogether, our results demonstrate that reward potentiates reinforcement-based adjustments in motor commands and that this effect persists even after reward removal on the subsequent day. The data also show that this effect does not concern all components of the movement, but specifically the amplitude of the force which was the most relevant factor for task success.

\section{DISCUSSION}

In this study, we investigated the net effect of reward on motor learning while controlling for the reinforcement feedback received by the participants. Our results provide evidence that reward can improve motor skill learning and that this effect is related to a specific potentiation of reinforcement-related adjustments in motor commands. Strikingly, the potentiation of such adjustments persisted on a subsequent day in the absence of reward. Moreover, such boosting of reinforcement-based adjustments did not concern all components of force production but only the amplitude, which was the most relevant one for task success. These findings shed light on the mechanisms through which reward can durably enhance motor performance. They also lay the groundwork for future rehabilitation strategies involving optimized sensory and reinforcement feedbacks.

A main goal of the present study was to explore the net effect of reward on motor skill learning by experimentally dissociating it from the reinforcement feedback. As such, previous motor learning studies have often coupled reinforcement and reward (e.g., Abe et al., 2011; Steel et al., 2019, 2016; Wachter et al., 2009; Wilkinson et al., 2015), based on the underlying assumption that receiving knowledge of performance (e.g., points or binary feedback) provides a form of intrinsic reward that can by itself increase motivation to perform well (Leow et al., 2018). However, in addition to providing some form of intrinsic reward, reinforcement feedback also provides a learning signal to the motor system that can influence motor learning (Bernardi et al., 2015; Galea et al., 2015; Huang et al., 2011; Kim et al., 2019; Leow et al., 2018; Mawase et al., 2017; Nikooyan et al., 2015; Shmuelof et al., 2012; Therrien et al., 2016; Uehara et al., 2018). In order to assess the net effect of motivation on motor learning, we therefore compared groups of participants trained with different monetary rewards but with the exact same reinforcement feedback. We found that motivation by reward allowed marked improvements in motor performance that were maintained after reward removal and even $24 \mathrm{~h}$ later (Figure 2). Notably, this was the case despite the fact that reinforcement alone was not sufficient to influence motor learning in our task. This demonstrates that the motivational context experienced during training can by itself strongly influence motor skill learning beyond performance-based reinforcement feedback.

The prospect of obtaining rewards for good performance enhances motivation but does not provide any additional learning signal to the motor system (Berke, 2018). Yet, it may boost the reliance on sensory and/or reinforcement feedbacks (Kim et al., 2019). To explore this possibility, we developed an analysis allowing us to investigate how participants adjusted their motor commands based on sensory or reinforcement feedbacks while controlling for differences in performance between the groups (see STAR Methods for more details). Interestingly, we found that reward specifically boosted reinforcement-based adjustments, following both positive and negative feedbacks, while sensory-based adjustments remained unaffected by reward (Figure 3). This suggests that reward boosted both the reproduction of successful behavior (exploitation) and correction of motor commands after failure (exploration; Dhawale et al., 2017). This was the case despite the fact that both types of feedback were relevant to improve motor performance at the single-trial level (Figures 3B, 3D, 3F, 3H). This result suggests that reward increases the reliance on reinforcement information during the learning process, with less effect on sensory-based adjustments. Interestingly, this finding may explain why tasks that strongly emphasize sensory-based learning (over reinforcement-based learning; Cashaback et al., 2017; Izawa and Shadmehr, 2011), often show less sensitivity to motivation. Accordingly, monetary reward shows little impact on sensorimotor adaptation (Galea et al., 2015; Hill et al., 2020) and on motor skill acquisition in tasks that strongly rely on sensory feedback (e.g., Abe et al., 2011; Steel et al., 2016; Widmer et al., 2016). The differential effect of reward on sensory and reinforcement-based adjustments may be due to the qualitatively different learning processes that are driven by these two types of feedbacks (Cashaback et al., 2017; Uehara et al., 2018). As such, while sensory feedback promotes error correction by providing directional feedback (Shadmehr et al., 2010), reinforcement can guide motor exploration based on binary feedback about task success (Therrien et al., 2016). Our results, along with the observation that monetary rewards are less effective in tasks where learning is dominated by sensory feedback, suggest that the potential of reward to improve motor learning relies on the boosting of a 
reinforcement learning mechanism. Based on this, we propose that the susceptibility of a given motor learning task to reward may depend on the relative contribution of sensory and reinforcement feedbacks in the learning process. Characterizing what type of motor tasks can benefit from motivational interventions is an important line of future work to translate fundamental motor control research into innovative rehabilitation procedures.

The finding of a reward-dependent boosting of reinforcement-based adjustments is in line with previous neuroimaging results showing that reward increases reinforcement-related activity in the striatum in the context of motor learning (Widmer et al., 2016). This reward-driven increase in striatal activity is reduced after a stroke (even when the striatum is unlesioned), a process that may contribute to the motor learning deficits observed in these patients (Widmer et al., 2019). Moreover, such reward-dependent modulation of motor adjustments has been shown to rely on dopamine (Galea et al., 2013; Pekny et al., 2015), a key neurotransmitter of the striatal circuitry. Based on these elements and on the causal role of the striatum in reinforcement-based adjustments in motor commands (Nakamura and Hikosaka, 2006; Williams and Eskandar, 2006), we suspect that this region may be crucial for the beneficial effect of reward observed in the present study. Notably, the cerebellum (Carta et al., 2019; Heffley et al., 2018; Sendhilnathan et al., 2020; Vassiliadis et al., 2019; Wagner et al., 2017) and frontal areas (Dayan et al, 2014, 2018; Hamel et al., 2018; Palidis et al., 2019; Ramakrishnan et al., 2017; Sidarta et al., 2016) are also likely to contribute to reward-based motor learning. Further investigations are required to better delineate the neurophysiological bases of reward-related improvements in motor learning.

The beneficial effect of reward on single-trial adjustments was maintained on Day 2, even after reward removal. As in Day 1 training, reinforcement-based adjustments were boosted while sensory-based adjustments remained unchanged by reward. This persistent change in the specific reaction to the reinforcement feedback after reward removal is suggestive of an associative learning process. In associative learning, presentation of a neutral stimulus (i.e., a conditioned stimulus) that has been consistently paired with a rewarding stimulus (i.e., an unconditioned stimulus) during a training period elicits a behavior that was initially only generated in reaction to the reward (Pavlov, 1927; Rescorla and Wagner, 1972). Following this framework, it is possible that the repetitive pairing of the reinforcement feedback with the reward during training induced an implicit association between the two events that remained evident when the reward was removed. This could explain why strong reinforcement-specific adjustments were maintained on Day 2 in the reward group, even though no rewards were at stake anymore. Such associative learning processes are known to strongly influence autonomic responses (Pool et al., 2019), inhibitory control (Avraham et al., 2020; Lindström et al., 2019; Verbruggen et al., 2014), decision making (Lindström et al., 2019), and even sensorimotor adaptation (Avraham et al., 2020) in humans. We propose that associative learning may also contribute to the durable influence of motivation on motor skill learning (Abe et al., 2011; Sporn et al., 2020).

In order to better characterize the effect of reward on motor learning, we considered separately the different components of the movement and found that force amplitude was the most strongly affected, while the speed of initiation and force variability remained largely insensitive to reward. This suggests that reward can have a selective influence on the regulation of a specific component of motor control. Importantly, an estimation of the respective influence of each motor component on task success also showed that force amplitude was the most relevant component for the task. Notably, the specificity of the effect of reward on the regulation of one motor component is in accordance with the idea that multidimensional motor tasks (i.e., requiring the control of multiple motor components) can be decomposed in subtasks that are learned separately in the motor system (Ghahramani and Wolpert, 1997) In this framework, learning of the different motor components may depend on their respective relevance for task success (Ghahramani and Wolpert, 1997; van der Kooij et al., 2021). Such task relevance may be estimated based on a priori knowledge of the task (e.g., following instructions; Popp et al., 2020) and through the reliance on a credit assignment system allowing to estimate the particular influence of each motor component on task success through trial and error (McDougle et al., 2016; Parvin et al., 2018). Based on this idea, we believe that the strong relationship between the amplitude of the force and task success in the present task pushed participants of the reward group to largely modulate this component based on the reinforcement feedback. If this is the case, this would suggest that it is possible to affect the training of specific motor abilities by modulating the weight of individual motor components in the computation of the reinforcement feedback, an aspect that could be exploited in future rehabilitation protocols. Alternatively, reward might have specifically modulated the amplitude of the force independently of the relevance of this parameter. Although the present study cannot rule out this hypothesis, we believe that such interpretation is unlikely given previous demonstration that reward can improve several aspects of motor control concomitantly (Codol et al., 2020; Manohar et al., 2015). Another possibility is that reinforcement feedback alone was sufficient to maximally modulate initiation 
time and variability in this task, precluding us from observing a difference with the reward-based training because of some form of ceiling effect. Further studies are required to disentangle these potentially co-existing interpretations to guide the development of component-specific rehabilitation therapies (Norman et al., 2017).

\section{Limitations of the study}

Our findings suggest that extrinsic reward can improve the acquisition and maintenance of a motor skill by boosting reinforcement-based adjustments in motor commands. However, it should be noted that here we focused on a very simple unimanual task in which performance relied on the ability to modulate a 1-degree of freedom force. While our analysis of the different motor components suggests that reward may also improve the learning of more complex tasks (by selectively boosting the adjustment of the most relevant dimensions for task success), future studies should address the generalizability of our results by using tasks engaging more complex skills.

Besides, our single-trial analysis suggests that reward affects differently sensory and reinforcement-based adjustments in motor commands. Yet, sensory and reinforcement feedbacks were always coupled in the present task. We did so on purpose to avoid inducing conflict in the learning process, yet the reward effect we report here could be influenced by the relationship between these feedbacks. Hence, follow-up investigations should assess the effect of reward on sensory and reinforcement-based adjustments in situations where both feedback types are dissociated (Cashaback et al., 2017).

\section{STAR $\star$ METHODS}

Detailed methods are provided in the online version of this paper and include the following:

- KEY RESOURCES TABLE

- RESOURCE AVAILABILITY

O Lead contact

O Materials availability

O Data and code availability

- EXPERIMENTAL MODEL AND SUBJECT DETAILS

- METHOD DETAILS

O General aspects

O Sensory feedback

O Reinforcement feedback

O Experimental procedure

- QUANTIFICATION AND STATISTICAL ANALYSIS

O Motor skill learning and maintenance

O Between-trial adjustments in motor commands

Group features, muscle fatigue and monetary gains

\section{ACKNOWLEDGMENTS}

We would like to thank Benvenuto Jacob and Julien Lambert for helping with the development of the task and Wanda Materne for assistance with data acquisition. P.V. was a PhD student supported by the Fund for Research training in Industry and Agriculture (FRIA/FNRS; FC29690), and grants by the Platform for Education and Talent (Gustave Boël - Sofina Fellowships) and Wallonie-Bruxelles International. G.D. was a postdoctoral fellow supported by the Belgian National Funds for Scientific Research (FNRS, 1B134.18). F.C.H. was supported by the Defitech Foundation (Morges, CH). J.D. was supported by grants from the Belgian FNRS (F.4512.14) and the Fondation Médicale Reine Elisabeth (FMRE).

\section{AUTHOR CONTRIBUTIONS}

Pierre Vassiliadis: Conceptualization, Methodology, Formal Analysis, Investigation, Data Curation, Writing - Original Draft, Writing - Review \& Editing, Visualization, Funding acquisition.

Gerard Derosiere: Conceptualization, Methodology, Writing - Review \& Editing.

Cecile Dubuc: Methodology, Investigation, Data Curation, Writing - Review \& Editing. 
Aegryan Lete: Investigation, Data Curation, Writing - Review \& Editing.

Frederic Crevecoeur: Conceptualization, Writing - Review \& Editing.

Friedhelm C. Hummel: Conceptualization, Writing - Review \& Editing, Supervision.

Julie Duque: Conceptualization, Methodology, Writing - Review \& Editing, Supervision, Funding

acquisition.

\section{DECLARATION OF INTERESTS}

The authors declare no conflict of interest.

Received: April 29, 2021

Revised: June 16, 2021

Accepted: July 2, 2021

Published: July 23, 2021

\section{REFERENCES}

Abe, M., Schambra, H., Wassermann, E.M. Luckenbaugh, D., Schweighofer, N., and Cohen, L.G. (2011). Reward improves long-term retention of a motor memory through induction of offline memory gains. Curr. Biol. 21, 557-562. https:// doi.org/10.1016/j.cub.2011.02.030.

Avraham, G., Taylor, J.A., Ivry, R.B., and McDougle, S.D. (2020). An associative learning account of sensorimotor adaptation. bioRxiv. https://doi.org/10.1101/2020.09.14.297143.

Balleine, B.W., and O'Doherty, J.P. (2010). Human and rodent homologies in action control: corticostriatal determinants of goal-directed and habitual action. Neuropsychopharmacology 35 , 48-69. https://doi.org/10.1038/npp.2009.131.

Barron, A.B., Søvik, E., and Cornish, J.L. (2010). The roles of dopamine and related compounds in reward-seeking behavior across animal phyla. Front. Behav.Neurosci. 4, 1-9. https://doi.org/10. 3389/fnbeh.2010.00163.

Berke, J.D. (2018). What does dopamine mean? Nat. Neurosci. 21, 787-793. https://doi.org/10. 1038/s41593-018-0152-y.

Bernardi, N.F., Darainy, M., and Ostry, D.J. (2015). Somatosensory contribution to the initial stages of human motor learning. J. Neurosci. 35, $14316-$ 14326. https://doi.org/10.1523/JNEUROSCI. 1344-15.2015.

Brainard, D.H. (1997). The psychophysics toolbox. Spat. Vis. 10, 433-436. https://doi.org/10.1163/ $156856897 \times 00357$.

Bush, G., Vogt, B.A., Holmes, J., Dale, A.M., Greve, D., Jenike, M.A., and Rosen, B.R. (2002). Dorsal anterior cingulate cortex: a role in rewardbased decision making. Proc. Natl. Acad. Sci. U. S. A. 99, 523-528. https://doi.org/10.1073/ pnas.012470999.

Carta, I., Chen, C.H., Schott, A.L., Dorizan, S., and Khodakhah, K. (2019). Cerebellar modulation of the reward circuitry and social behavior. Science 80, 363. https://doi.org/10.1126/science.aav0581.

Cashaback, J.G.A., McGregor, H.R., Mohatarem, A., and Gribble, P.L. (2017). Dissociating error- based and reinforcement-based loss functions during sensorimotor learning. PLoS Comput. Biol. 13, 1-28. https://doi.org/10.1371/journal. pcbi.1005623.

Chen, X., Holland, P., and Galea, J.M. (2017). The effects of reward and punishment on motor skill learning. Curr.Opin.Behav. Sci. 20, 83-88. https:// doi.org/10.1016/j.cobeha.2017.11.011.

Codol, O., Holland, P.J., Manohar, S.G., and Galea, J.M. (2020). Reward-based improvements in motor control are driven by multiple error-reducing mechanisms. J. Neurosci. 40, 3604-3620. https://doi.org/10.1523/JNEUROSCl. 2646-19.2020.

Connell, L.A. (2008). Somatosensory impairment after stroke: frequency of different deficits and their recovery. https://doi.org/10.1177/ 0269215508090674.

Dayan, E., Hamann, X.J.M., Averbeck, B.B., and Cohen, L.G. (2014). Brain structural substrates of reward dependence during. Behav. Perform. 34 16433-16441. https://doi.org/10.1523/ JNEUROSCI.3141-14.2014.

Dayan, E., Herszage, J., Laor-Maayany, R., Sharon, H., and Censor, N. (2018).

Neuromodulation of reinforced skill learning reveals the causal function of prefrontal cortex Hum. Brain Mapp. 39, 4724-4732. https://doi. org/10.1002/hbm.24317.

Dayan, P., and Niv, Y. (2008). Reinforcement learning: the good, the bad and the ugly. Curr.Opin.Neurobiol. 18, 185-196. https://doi. org/10.1016/j.conb.2008.08.003.

Derosière, G., Alexandre, F., Bourdillon, N., Mandrick, K., Ward, T.E., and Perrey, S. (2014). Similar scaling of contralateral and ipsilateral cortical responses during graded unimanual force generation. Neuroimage 85, 471-477. https://doi.org/10.1016/j.neuroimage.2013.02 006.

Derosiere, G., and Perrey, S. (2012). Relationship between submaximal handgrip muscle force and NIRS-measured motor cortical activation. Adv.
Exp. Med. Biol. 737, 269-274. https://doi.org/10 1007/978-1-4614-1566-4_40.

Derosiere, G., Vassiliadis, P., Demaret, S., Zénon, A., and Duque, J. (2017a). Learning stagedependent effect of M1 disruption on value based motor decisions. Neuroimage 162, 173-185. https://doi.org/10.1016/j.neuroimage. 2017.08.075

Derosiere, G., Zénon, A., Alamia, A., and Duque, J. (2017b). Primary motor cortex contributes to the implementation of implicit value-based rules during motor decisions. Neuroimage 146, 11151127. https://doi.org/10.1016/j.neuroimage. 2016.10.010.

Dhawale, A.K., and Smith, M.A. (2017). The Role of Variability in Motor Learning.

Dhawale, A.K., Smith, M.A., and Ölveczky, B.P. (2017). The role of variability in motor learning. Annu. Rev. Neurosci. 40, 479-498. https://doi. org/10.1146/annurev-neuro-072116-031548.

Galea, J.M., Mallia, E., Rothwell, J., and Diedrichsen, J. (2015). The dissociable effects of punishment and reward on motor learning. Nat. Neurosci. 18, 597-602. https://doi.org/10.1038/ nn.3956.

Galea, J.M., Ruge, D., Buijink, A., Bestmann, S., and Rothwell, J.C. (2013). Punishment-induced behavioral and neurophysiological variability reveals dopamine-dependent selection of kinematic movement parameters. J. Neurosci. 33, 3981-3988. https://doi.org/10.1523/JNEUROSCl. 1294-12.2013.

Gershman, S.J., and Daw, N.D. (2017) Reinforcement Learning and Episodic Memory in Humans and Animals : An Integrative Framework. https://doi.org/10.1146/annurev-psych-122414. 033625 .

Ghahramani, Z., and Wolpert, D.M. (1997). Modular decomposition in visuomotor learning Nature 386, 392-395. https://doi.org/10.1038/ $386392 \mathrm{aO}$.

Grogan, J.P., Sandhu, T.R., Hu, M.T., and Manohar, S.G. (2020a). Dopamine promotes 
instrumental motivation, but reduces rewardrelated vigour. bioRxiv 9, e58321. https://doi.org/ 10.1101/2020.03.30.010074.

Grogan, John P., Sandhu, T.R., Hu, M.T., and Manohar, S.G. (2020b). Dopamine promotes instrumental motivation, but reduces rewardrelated vigour. Elife 9, 1-20. https://doi.org/10. 7554 /eLife.58321.

Hamel, R., Savoie, F.A., Lacroix, A., Whittingstall, K., Trempe, M., and Bernier, P.M. (2018). Added value of money on motor performance feedback: increased left central beta-band power for rewards and fronto-central theta-band power for punishments. Neuroimage 179, 63-78. https:// doi.org/10.1016/j.neuroimage.2018.06.032.

Hare, T.A., Schultz, W., Camerer, C.F., O'Doherty, J.P., and Rangel, A. (2011). Transformation of stimulus value signals into motor commands during simple choice. Proc. Natl. Acad. Sci. U. S. A. 108, 18120-18125. https://doi.org/10 1073/pnas.1109322108.

Heffley, W., Song, E.Y., Xu, Z., Taylor, B.N., Hughes, M.A., McKinney, A., Joshua, M., and Hull, C. (2018). Coordinated cerebellar climbing fiber activity signals learned sensorimotor predictions. Nat. Neurosci. 21, 1431-1441. https://doi.org/10.1038/s41593-018-0228-8.

Hepworth, L.R., Rowe, F.J., Walker, M.F., and Rockliffe, J. (2016). Post-stroke Visual Impairment: A Systematic Literature Review of Types and Recovery of Visual Conditions Poststroke Visual Impairment : A Systematic Literature Review of Types and Recovery of Visual Conditions. https://doi.org/10.9734/OR/2016/ 21767.

Hill, C.M., Stringer, M., Waddell, D.E., and Del Arco, A. (2020). Punishment feedback impairs memory and changes cortical feedback-related potentials during motor learning. Front. Hum. Neurosci. 14,1-14. https://doi.org/10.3389/ fnhum 2020.00294

Holland, P., Codol, X, Oxley, E., Taylor, M. Hamshere, E., Joseph, S., Huffer, L., and Gale, X.M. (2019). Domain-specific working memory, but not dopamine-related genetic variability, shapes reward-based motor learning. J. Neurosci. 39, 9383-9396. https://doi.org/10. 1523/JNEUROSCI.0583-19.2019.

Huang, V.S., Haith, A., Mazzoni, P., and Krakauer, J.W. (2011). Rethinking motor learning and savings in adaptation paradigms: model-free memory for successful actions combines with internal models. Neuron 70, 787-801. https://doi. org/10.1016/j.neuron.2011.04.012.

Izawa, J., and Shadmehr, R. (2011). Learning from sensory and reward prediction errors during motor adaptation. PLoS Comput. Biol. 7, 1-12. https://doi.org/10.1371/journal.pcbi.1002012.

Kim, H.E., Parvin, D.E., and Ivry, R.B. (2019). The influence of task outcome on implicit motor learning. Elife 8, 1-28. https://doi.org/10.7554/ eLife.39882.

Krakauer, J.W., Hadjiosif, A.M., Xu, J., Wong, A.L., and Haith, A.M. (2019). Motor learning. Compr. Physiol. 9, 613-663. https://doi.org/10.1002/ cphy.c170043.
Lardi, C., Billieux, J., D'Acremont, M., and Van derLinden, M. (2008). A French adaptation of a short version of the sensitivity to punishment and sensitivity to reward questionnaire (SPSRQ). Pers. Individ. Dif. 45, 722-725. https://doi.org/10.1016/ j.paid.2008.07.019

Leow, L.A., Marinovic, W., de Rugy, A., and Carroll, T.J. (2018). Task errors contribute to implicit aftereffects in sensorimotor adaptation. Eur. J. Neurosci. 48, 3397-3409. https://doi.org/ 10.1111/ejn.14213.

Levy, S., Lavzin, M., Benisty, H., Ghanayim, A., Dubin, U., Achvat, S., Brosh, Z., Aeed, F., Mensh, B.D., Schiller, Y., et al. (2020). Cell-type-specific outcome representation in the primary motor cortex. Neuron 107, 954-971.e9. https://doi.org/ 10.1016/j.neuron.2020.06.006.

Lewthwaite, R., and Wulf, G. (2017). Optimizing motivation and attention for motor performance and learning. Curr. Opin. Psychol. 16, 38-42. https://doi.org/10.1016/j.copsyc.2017.04.005.

Lindström, B., Golkar, A., Jangard, S., Tobler, P.N., and Olsson, A. (2019). Social threat learning transfers to decision making in humans. Proc. Natl. Acad. Sci. U. S. A. 116, 4732-4737. https:// doi.org/10.1073/pnas.1810180116.

Manohar, S.G., Chong, T.T., Apps, M.A.J., Jarman, P.R., Bhatia, K.P., Husain, M., Manohar, S.G., Chong, T.T., Apps, M.A.J., Batla, A., and Stamelou, M. (2015). Reward pays the cost of noise reduction in motor and cognitive control article reward pays the cost of noise reduction in motor and cognitive control. Curr. Biol. 25, 1707 1716. https://doi.org/10.1016/j.cub.2015.05.038.

Mawase, F., Uehara, S., Bastian, A.J., and Celnik P. (2017). Motor learning enhances usedependent plasticity. J. Neurosci. 37, 2673-2685. https://doi.org/10.1523/JNEUROSCI.3303-16. 2017

McDougle, S.D., Boggess, M.J., Crossley, M.J., Parvin, D., Ivry, R.B., and Taylor, J.A. (2016). Credit assignment in movement-dependent reinforcement learning. Proc. Natl. Acad. Sci. U. S. A. 113, 6797-6802. https://doi.org/10.1073/ pnas. 1523669113.

McGrane, N., Galvin, R., Cusack, T., and Stokes, E. (2015). Addition of motivational interventions to exercise and traditional physiotherapy: a review and meta-analysis. Physiother. (United Kingdom) 101, 1-12. https://doi.org/10.1016/j.physio.2014 04.009.

Nakamura, K., and Hikosaka, O. (2006) Facilitation of saccadic eye movements by postsaccadic electrical stimulation in the primate caudate. J. Neurosci. 26, 12885-12895. https:// doi.org/10.1523/JNEUROSCI.3688-06.2006.

Nikooyan, A.A., Ahmed, A.A., Nikooyan, A.A., and Ahmed, A.A. (2015). Reward feedback accelerates motor learning. J. Neurphysiol. 113, 633-646. https://doi.org/10.1152/jn.00032.2014.

Norman, S.L., Lobo-Prat, J., and Reinkensmeyer, D.J. (2017). How do strength and coordination recovery interact after stroke? A computational model for informing robotic training. IEEE Int. Conf. Rehabil. Robot. 181-186. https://doi.org/ 10.1109/ICORR.2017.8009243.
O'Doherty, J.P. (2004). Reward representations and reward-related learning in the human brain: Insights from neuroimaging. Curr.Opin.Neurobiol. 14, 769-776. https://doi. org/10.1016/j.conb.2004.10.016.

Oldfield, R.C. (1971). The assessment and analysis of handedness: the Edinburgh inventory. Neuropsychologia 9, 97-113. https://doi.org/10. 1016/0028-3932(71)90067-4

Padoa-Schioppa, C. (2011). Neurobiology of economic choice: a good-based model. Annu. Rev. Neurosci. 34, 333-359. https://doi.org/10 1146/annurev-neuro-061010-113648.

Palidis, D.J., Cashaback, J.G.A., and Gribble, P.L. (2019). Neural signatures of reward and sensory error feedback processing in motor learning. J. Neurophysiol. 121, 1561-1574. https://doi.org/ 10.1152/jn.00792.2018

Palminteri, S., Lebreton, M., Worbe, Y., Hartmann, A., Lehéricy, S., Vidailhet, M., Grabli, D., and Pessiglione, M. (2011). Dopaminedependent reinforcement of motor skill learning: evidence from Gilles de la Tourette syndrome. Brain 134, 2287-2301. https://doi.org/10.1093/ brain/awr147.

Parvin, D.E., McDougle, S.D., Taylor, J.A., and Ivry, R.B. (2018). Credit assignment in a motor decision making task is influenced by agency and not sensorimotor prediction errors. J. Neurosci. 38, 3601-3617. https://doi.org/10.1523/ JNEUROSCI.3601-17.2018.

Pavlov, I. (1927). Conditioned Reflexes: An Investigation of the Physiological Activity of the Cerebral Cortex (Oxford Univ. Press).

Pekny, S.E., Izawa, J., and Shadmehr, R. (2015). Reward-dependent modulation of movement variability. J. Neurosci. 35, 4015-4024. https://doi. org/10.1523/JNEUROSCI.3244-14.2015.

Pelli, D.G. (1997). The VideoToolbox software for visual psychophysics: transforming numbers into movies. Spat. Vis. 10,437-442. https://doi.org/10. $1163 / 156856897 \times 00366$

Pool, E.R., Pauli, W.M., Kress, C.S., and O'Doherty, J.P. (2019). Behavioural evidence for parallel outcome-sensitive and outcomeinsensitive Pavlovian learning systems in humans. Nat. Hum. Behav. 3, 284-296. https://doi.org/10 1038/s41562-018-0527-9.

Popp, N.J., Yokoi, A., Gribble, P.L., and Diedrichsen, J. (2020). The effect of instruction on motor skill learning. J. Neurophysiol. 124, 14491457. https://doi.org/10.1152/JN.00271.2020.

Quattrocchi, G., Greenwood, R., Rothwell, J.C., Galea, J.M., and Bestmann, S. (2017). Reward and punishment enhance motor adaptation in stroke. J. Neurol. Neurosurg. Psychiatry 88, 730-736. https://doi.org/10.1136/jnnp-2016-314728.

Ramakrishnan, A., Byun, Y.W., Rand, K., Pedersen, C.E., Lebedev, M.A., and Nicolelis, M.A.L. (2017). Cortical neurons multiplex reward-related signals along with sensory and motor information. Proc. Natl. Acad. Sci. U. S. A. 114, E4841-E4850. https://doi.org/10.1073/pnas.1703668114.

Ramkumar, P., Dekleva, B., Cooler, S., Miller, L., and Kording, K. (2016). Premotor and motor 
cortices encode reward. PLoS One 11, 1-13. https://doi.org/10.1371/journal.pone.0160851.

Raspopovic, S., Capogrosso, M., Petrini, F.M. Bonizzato, M., Rigosa, J., Pino, G.D., Carpaneto, J., Controzzi, M., Boretius, T., Fernandez, E., et al. (2014). Restoring natural sensory feedback in realtime bidirectional hand prostheses. Sci. Transl. Med. 6, 222ra19.

Rescorla, R., and Wagner, A. (1972). A theory of Pavlovian conditioning: Variations in the effectiveness of reinforcement and nonreinforcement. In Classical Conditioning II: Current Research. And Theory, A.H. Black and W.F. Prokasy, eds., pp. 64-99.

Roemmich, R.T., and Bastian, A.J. (2018). Closing the loop: from motor neuroscience to neurorehabilitation. Annu. Rev. Neurosci. 41, 415-429. https://doi.org/10.1146/annurev-neuro080317-062245.

Schultz, W. (2015). Neuronal reward and decision signals: from theories to data. Physiol. Rev. 95 , 853-951. https://doi.org/10.1152/physrev.00023. 2014.

Sendhilnathan, N., Ipata, A.E., and Goldberg, M.E. (2020). Neural correlates of reinforcement learning in mid-lateral cerebellum. Neuron 106 188-198.e5. https://doi.org/10.1016/j.neuron 2019.12.032.

Shadmehr, R., Reppert, T.R., Summerside, E.M. Yoon, T., and Ahmed, A.A. (2019). Movement vigor as a Re fl ection of subjective economic utility. Trends Neurosci. xx, 1-14. https://doi.org/ 10.1016/j.tins.2019.02.003.

Shadmehr, R., Smith, M.A., and Krakauer, J.W. (2010). Error Correction, Sensory Prediction, and Adaptation in Motor Control. https://doi.org/10. 1146/annurev-neuro-060909-153135.

Shima, K., and Tanji, J. (1998). Role for cingulate motor area cells in voluntary movement selection based on reward. Science 282, 1335-1338. https://doi.org/10.1126/science.282.5392.1335.

Shmuelof, L., Huang, V.S., Haith, A.M., Delnicki, R.J., Mazzoni, P., and Krakauer, J.W. (2012). Overcoming motor "forgetting" through

reinforcement of learned actions. J. Neurosci. 32, 14617-14621a. https://doi.org/10.1523/ JNEUROSCI.2184-12.2012.

Sidarta, A., Vahdat, S., Bernardi, N.F., and Ostry, D.J. (2016). Somatic and reinforcement-based plasticity in the initial stages of human motor learning. J. Neurosci. 36, 11682-11692. https:// doi.org/10.1523/JNEUROSCI.1767-16.2016.
Sidarta, X.A., Van Vugt, X.F.T., and Ostry, D.J. (2019). Control of Movement Somatosensory working memory in human reinforcement-based motor learning. J. Neurophysiol. 120, 3275-3286. https://doi.org/10.1152/in.00442.2018.

Sporn, S., Chen, X., and Galea, J.M. (2020) Reward-based invigoration of sequential reaching. bioRxiv, 1-38. https://doi.org/10.1101/ 2020.06.15.152876.

Steel, A., Silson, E.H., Stagg, C.J., and Baker, C.I (2019). Differential impact of reward and punishment on functional connectivity after skill learning. Neuroimage 189, 95-105. https://doi. org/10.1016/j. neuroimage.2019.01.009.

Steel, A., Silson, E.H., Stagg, C.J., and Baker, C.I (2016). The impact of reward and punishment on skill learning depends on task demands. Sci. Rep. 6, 1-9. https://doi.org/10.1038/srep36056.

Therrien, A.S., Wolpert, D.M., and Bastian, A.J. (2016). Effective reinforcement learning following cerebellar damage requires a balance between exploration and motor noise. Brain 139, 101-114. https://doi.org/10.1093/brain/awv329.

Torrubia, R., Ávila, C., Moltó, J., and Caseras, X. (2001). The sensitivity to punishment and sensitivity to reward questionnaire (SPSRQ) as a measure of Gray's anxiety and impulsivity dimensions. Pers. Individ. Dif. 31, 837-862. https://doi.org/10.1016/S0191-8869(00)00183-5.

Tseng, Y.-W., Diedrichsen, J., Krakauer, J.W Shadmehr, R., and Bastian, A.J. (2007). Sensory prediction errors drive cerebellum-dependent adaptation of reaching. J. Neurophysiol. 98 54-62. https://doi.org/10.1152/jn.00266.2007.

Uehara, S., Mawase, F., and Celnik, P. (2018). Learning similar actions by reinforcement or sensory-prediction errors rely on distinct physiological mechanisms. Cereb.Cortex 28, 3478-3490. https://doi.org/10.1093/cercor/ bh $\times 214$.

Uehara, S., Mawase, F., Therrien, A.S., Cherryallen, K.M., Celnik, P., and Hopkins, J. (2019) Interactions between Motor Exploration and Reinforcement Learning.

van der Kooij, K., van Mastrigt, N.M., Crowe, E.M. and Smeets, J.B.J. (2021). Learning a reach trajectory based on binary reward feedback. Sci. Rep. 11, 1-15. https://doi.org/10.1038/s41598020-80155-x.

Vassiliadis, P., and Derosiere, G. (2020). Selecting and executing actions for rewards. J. Neurosci. 40, 6474-6476. https://doi.org/10.1523/ JNEUROSCI.1250-20.2020
Vassiliadis, P., Derosiere, G., and Duque, J. (2019). Beyond motor noise : considering other causes of impaired reinforcement learning in cerebellar patients. eNeuro 6, 1-4. https://doi. org/10.1523/ENEURO.0458-18.2019.

Verbruggen, F., Best, M., Bowditch, W.A. Stevens, T., and McLaren, I.P.L. (2014). The inhibitory control reflex. Neuropsychologia 65 263-278. https://doi.org/10.1016/1. neuropsychologia.2014.08.014.

Wachter, T., Lungu, O.V., Liu, T., Willingham, D.T., and Ashe, J. (2009). Differential effect of reward and punishment on procedural learning. J. Neurosci. 29, 436-443. https://doi.org/10.1523/ JNEUROSCI.4132-08.2009.

Wagner, M.J., Kim, T.H., Savall, J., Schnitzer, M.J., and Luo, L. (2017). Cerebellar granule cells encode the expectation of reward. Nature 544, 96-100. https://doi.org/10.1038/nature21726.

Widmer, M., Lutz, K., and Luft, A.R. (2019) Reduced striatal activation in response to rewarding motor performance feedback after stroke. Neuroimage Clin. 24, 102036. https://doi. org/10.1016/j.nicl.2019.102036.

Widmer, M., Ziegler, N., Held, J., Luft, A., and Lutz, K. (2016). Rewarding feedback promotes motor skill consolidation via striatal activity. Prog Brain Res. 229, 303-323. https://doi.org/10.1016/ bs.pbr.2016.05.006.

Wilkinson, L., Steel, A., Mooshagian, E. Zimmermann, T., Keisler, A., Lewis, J.D., and Wassermann, E.M. (2015). Online feedback enhances early consolidation of motor sequence learning and reverses recall deficit from transcranial stimulation of motor cortex. Cortex 71, 134-147. https://doi.org/10.1016/j.cortex.2015.06.012.

Williams, Z.M., and Eskandar, E.N. (2006). Selective enhancement of associative learning by microstimulation of the anterior caudate. Nat. Neurosci. 9, 562-568. https://doi.org/10.1038/ nn1662.

Wu, H.G., Miyamoto, Y.R., Castro, L.N.G., Ölveczky, B.P., and Smith, M.A. (2014). Temporal structure of motor variability is dynamically regulated and predicts motor learning ability. Nat. Neurosci. 17, 312-321. https://doi.org/10. 1038/nn.3616.

Yamazaki, Y., Hikishima, K., Saiki, M., Inada, M., Sasaki, E., Lemon, R.N., Price, C.J., Okano, H., and Iriki, A. (2016). Neural changes in the primate brain correlated with the evolution of complex motor skills. Nat. Publ. Gr. 6, 31084. https://doi.org/10.1038/ srep31084. 


\section{STAR $\star$ METHODS}

\section{KEY RESOURCES TABLE}

\begin{tabular}{|c|c|c|}
\hline REAGENT or RESOURCE & SOURCE & IDENTIFIER \\
\hline \multicolumn{3}{|l|}{ Deposited Data } \\
\hline Motor learning data ('All_Variables.mat') & This paper & https://osf.io/5pjem/ \\
\hline Subjects characteristics ('Subjects_characteristics.xlsx') & This paper & https://osf.io/5pjem/ \\
\hline \multicolumn{3}{|l|}{ Software and Algorithms } \\
\hline Matlab vR2007 7.5 and R2018a & Mathworks & www.mathworks.com/products/matlab.html \\
\hline Statistica 10 & StatSoft Inc. & https://www.statsoft.de/en/software/tibco-statisticatm \\
\hline Psychophysics Toolbox & Psychtoolox.org & http://psychtoolbox.org/ \\
\hline
\end{tabular}

\section{RESOURCE AVAILABILITY}

\section{Lead contact}

Further information and requests should be directed to the lead contact, Pierre Vassiliadis (pierre. vassiliadis@uclouvain.be).

\section{Materials availability}

This study did not generate new unique reagents.

\section{Data and code availability}

- Motor learning data ('All_Variables.mat') and de-identified subjects characteristics ('Subjects_ characteristics.xlsx') are freely available via an open-access data sharing repository (https://osf.io/ 5pjem/).

- This paper does not report original code.

- Any additional information required to reanalyze the data reported in this paper is available from the lead contact upon request.

\section{EXPERIMENTAL MODEL AND SUBJECT DETAILS}

A total of 90 right-handed healthy volunteers participated in the present study ( 58 women, $23.7 \pm 0.3$ years old; mean \pm SE). Handedness was determined via a shortened version of the Edinburgh Handedness Inventory (Oldfield, 1971). None of the participants suffered from any neurological or psychiatric disorder, nor were they taking any centrally-acting medication. All participants gave their written informed consent in accordance with the Ethics Committee of the Université Catholique de Louvain (approval number: 2018/ $22 \mathrm{MAl} / 219$ ) and the principles of the Declaration of Helsinki. Subjects were financially compensated for their participation. Finally, all participants were asked to fill out a French adaptation of the Sensitivity to Punishment and Sensitivity to Reward Questionnaire (SPSRQ; Lardi et al., 2008; Torrubia et al., 2001).

\section{METHOD DETAILS}

\section{General aspects}

Participants were seated approximately $60 \mathrm{~cm}$ in front of a computer screen (refresh rate $=100 \mathrm{~Hz}$ ) with their right forearm positioned at a right angle on the table. The task was developed on Matlab 7.5 (the Mathworks, Natick, Massachusetts, USA) exploiting the Psychophysics Toolbox extensions (Brainard, 1997; Pelli, 1997) and consisted in an adaptation of previously used motor learning tasks (Abe et al., 2011; Mawase et al., 2017; Steel et al., 2016). The task required participants to squeeze a force transducer (Arsalis, Belgium) between the index and the thumb to control the one-dimension motion of a cursor displayed on the screen. Increasing force resulted in the cursor moving vertically and upward. Each trial started with a preparatory phase in which a sidebar appeared at the bottom of the screen and a target at the top (Figure 1A). After a variable time interval, a cursor popped up in the sidebar and participants had to 
pinch the transducer to move the cursor as quickly as possible from the sidebar to the target and maintain it there for the rest of the task. The level of force required to reach the target (Target Force $_{\text {) was individualized }}$ for each participant and set at $10 \%$ of maximum voluntary contraction (MVC). Notably, squeezing the transducer before the appearance of the cursor was considered as anticipation and therefore led to an interruption of the trial. Such trials were discarded from further analyses. At the end of each trial, a binary reinforcement feedback represented by a colored circle was provided to the subject followed by a reminder of the color/feedback association and potential monetary reward associated with good performance (see Reinforcement feedback section below).

\section{Sensory feedback}

We provided only limited visual feedback to the participants (Mawase et al., 2017). As such, on most trials (90\%), the cursor disappeared shortly after the subject started to squeeze the force transducer (partial vision trials); it became invisible as soon as the generated force became larger than half of the Target Force (i.e., $5 \%$ of MVC). Conversely, the remaining trials (10\%) provided a continuous vision of the cursor (full vision trials). Therefore, on most trials, participants had limited visual information and had to rely exclusively

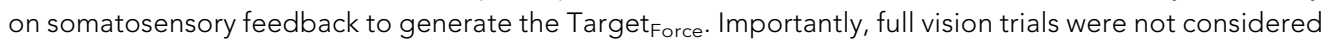
in the analyses.

\section{Reinforcement feedback}

At the end of each trial, subjects were presented with a binary reinforcement feedback indicating performance. Success on the task was determined based on the Error; that is, the absolute force difference be-

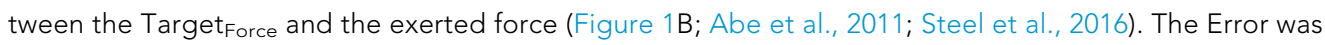
computed for each frame refresh (i.e., at $100 \mathrm{~Hz}$ ) from $150 \mathrm{~ms}$ to the end of the trial and then averaged for each trial (Steel et al., 2016) and expressed in percentage of MVC. This indicator of performance allowed us to classify a trial as successful or not based on an individualized success threshold (see below). When the Error on a given trial was below the threshold (negative normalized Error), the trial was considered as successful, and when it was above the threshold (positive normalized Error), the trial was considered as failed. Hence, task success depended on the ability to reduce the Error by approximating the Target $_{\text {Force }}$ as quickly and accurately as possible. Importantly, participants were told explicitly that both speed and accuracy were taken into account to determine task success. In summary, to be successful, participants knew that they had to quickly initiate the force and be as accurate as possible in reproducing the Target $_{\text {Force. }}$

In different blocks of trials, we manipulated the reinforcement feedback and reward provided during training. In Block_s, the reinforcement feedback was non-informative (magenta circle regardless of performance), and participants could only rely on somatosensory feedback to perform the task. In Block_sR, the reinforcement feedback consisted of a yellow (representing a successful trial) or blue circle (representing a failed trial), providing knowledge of performance (Figure 1A). In Block_sRR, this knowledge of performance was associated with a monetary reward (+8 cents or 0 cent for Success or Failure, respectively). Therefore, contrarily to Block_s, Block_sR and Block_sRR provided knowledge of performance and this feedback was associated with a monetary reward in Block_sRR.

\section{Experimental procedure}

Subjects' performance was tested for two consecutive days (Day 1 and Day 2; Figure 1C). On Day 1, we first

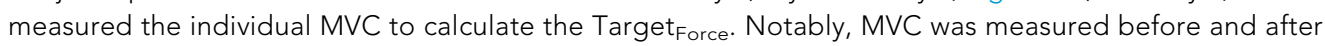
both sessions to assess potential muscle fatigue related to the training (see 4.4.3). Participants then performed 2 blocks of Familiarization. In the first block, participants performed 20 full vision trials; it served to familiarize the subjects with the task in a Block_sR setting (Full vision block). Subsequently, all blocks were composed of a mixture of full vision trials ( $10 \%$ of total trials) and partial vision trials ( $90 \%$ of total trials). The second Familiarization block consisted of 20 trials and allowed us to determine baseline performance to individualize the difficulty of the task for the rest of the experiment (Calibration block). For every subject, each partial vision trial of the Calibration block was classified in terms of Error from the lowest to the greatest in percentage of MVC. We took the $35^{\text {th }}$ percentile of the Error to determine the individual success threshold. Success thresholds were constrained between 2 and 3.5\% of MVC by asking participants to repeat the Calibration block when the computed threshold was outside these boundaries. Those parameters were determined based on pilot data to obtain coherent learning curves among individuals. 
After the Familiarization and Calibration blocks, the first experimental session consisted of 280 trials divided in 8 blocks. All subjects started with a Block_sR of 20 trials to evaluate the performance at Pretraining and similarly ended the session with a Post-training assessment of 20 trials. In between, 6 Training blocks of 40 trials were performed by the participants (Figure 1B). During this Training period, individuals were split into 3 separate groups (Group TYPE: Group_s, Group_sR or Group_sRR) depending on the type of blocks they performed during training. As such, Group_s completed Block_s, Group_sR performed Block SR and Group_sRR trained under Block_sRR condition. Contrasting performance in the Pre- and Post-training blocks allowed us to evaluate learning of the skill under the three training conditions. 24h later, subjects

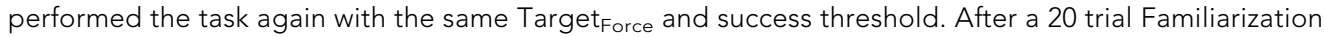
used to remind the task to participants, they performed 140 trials split in 4 blocks; all were performed in a Block_sR setting. This Re-test session allowed us to assess skill maintenance $24 \mathrm{~h}$ after training.

\section{QUANTIFICATION AND STATISTICAL ANALYSIS}

Statistical analyses were carried out with Matlab 2018a (the Mathworks, Natick, Massachusetts, USA) and Statistica 10 (StatSoft Inc., Tulsa, Oklahoma, USA). Post-hoc comparisons were always conducted using the Fisher's LSD procedure. The significance level was set at $p \leq 0.05$, except in the case of correction for multiple comparisons (see below).

\section{Motor skill learning and maintenance}

The main aim of the present study was to evaluate the effect of reward on motor skill learning and maintenance. To assess skill learning, we expressed the median Error at Post-training in percentage of the value obtained at Pre-training. To evaluate skill maintenance, we expressed the median Error during the Re-test session in percentage of Pre-training. First, we compared skill learning and maintenance between the groups through one-way ANOVAs with the factor GrouptYPE. Then, we explored the significance of skill learning and maintenance within each group by conducting Bonferroni-corrected single sample t-tests on these percentage data against a constant value of $100 \%$ (i.e., corresponding to the Pre-training level).

As explained above, task performance depended on both the speed and the accuracy of the produced force (Figure 1B). We characterized the effect of reward on these different levels of force control, by evaluating separately the speed of force initiation and the accuracy of the maintained force. To evaluate the speed of force

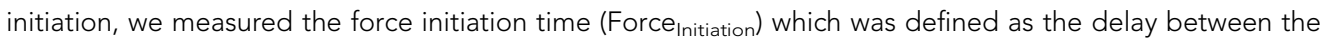
appearance of the cursor and the moment where the applied force reached 5\% of MVC (i.e., corresponding

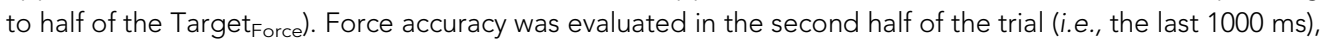
through two different parameters. First, we computed the Amplitude Error of the force (Force AmplError), defined as the absolute difference between the mean force exerted in the last $1000 \mathrm{~ms}$ of the trial and the Target $t_{\text {Force. }}$ It reflected how much the amplitude of the maintained force differed from the Target Force. $_{\text {Sec- }}$ ond, force accuracy was also characterized by considering the variability of the maintained force, with high levels of variability causing increases in the Error. To assess force variability (Forcevariability), we computed the coefficient of variation of the force in the second half of the trial (i.e., standard deviation of force/mean force). In summary, to be successful, participants had to quickly initiate the force (i.e., low Force Initiation $_{\text {) }}$ and be as accurate as possible (i.e., low Force AmplError $_{\text {and Force }}$ ariability).

As a control, we verified that the three motor components described above (i.e., Force Initiation, Force $_{\text {AmplError, }}$

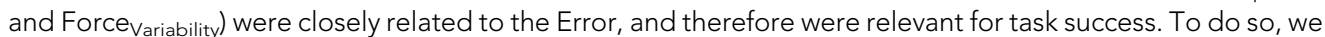
ran partial linear regressions on the Error data with Force $_{\text {Initiation, }}$ Force $_{\text {AmplError }}$ and Force Variability $_{\text {as predictors }}$ to estimate the respective influence of each motor component on the Error, while controlling for the effect of

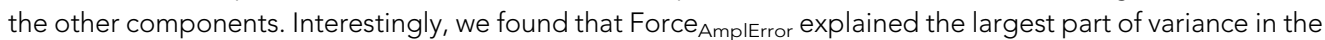
Error ( $r=0.96 \pm 0.003 ; p<0.05$ in 90/90 subjects). Force Initiation also explained a large part of variance in the

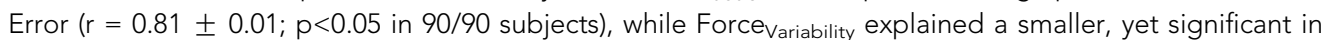
most subjects, part of variance $(r=0.22 \pm 0.03 ; p<0.05$ in 68/90 subjects). Hence, although all motor param-



\section{Between-trial adjustments in motor commands}

A second goal of the present study was to assess the effect of reward on between-trial adjustments in motor commands. Specifically, we aimed at evaluating how motor commands were adjusted based on reinforcement and sensory feedback in our three experimental groups. 
To do so, for each trial ${ }_{n}$ we computed the absolute between-trial change (BTC) in Error (Error BTC; $_{\text {i see (Pekny }}$ et al., 2015; Uehara et al., 2019) for similar approaches in reaching tasks).

$$
\text { BTC Error }=|\operatorname{Error}(\mathrm{n}+1)-\operatorname{Error}(\mathrm{n})|
$$

In order to study how much motor commands were adjusted based on previous experience, we compared adjustments in motor commands following trials of different Error magnitudes. To do so, we first subtracted each subject's individual success threshold to the Error data. Hence, normalized Errors below 0 corresponded to successful trials and normalized Errors above 0 corresponded to failed trials. Then, we split

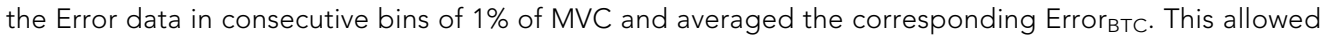
us to compare Error $_{\text {BTC }}$ following trials of similar Error ${ }_{n}$ across the groups.

As a first step, to better understand how motor commands were adjusted based on the reinforcement feedback, we compared Error ${ }_{\mathrm{BTC}}$ following bins of Success or Failure trials of neighboring Error magnitudes

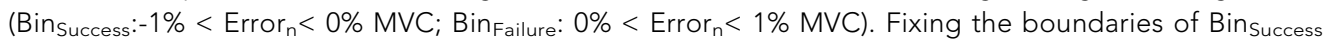
and $\mathrm{Bin}_{\text {Failure }}$ allowed us to compare reinforcement-related adjustments between the groups while controlling for the magnitude of Error ; an aspect that might directly influence between-trial adjustments. First, we performed a two-way ANOVA with the factors Outcome (Success or Failure) and GrouptYPE. We then computed reinforcement-based adjustments as the percentage change in Error $_{\text {BTC }}$ in Bin Failure compared to Binsuccess. This index allowed us to determine in a single measure how participants from the different groups adjusted their behavior based on the reinforcement obtained in the previous trial.

$$
\text { Reinforcement }- \text { based adjustments in } \text { Error }_{\text {BTC }}=100 \times \frac{\text { ErrorBTC }(\text { BinFailure })}{\text { ErrorBTC }(\text { BinSuccess })}
$$

These analyses were conducted separately on the Day 1 and Day 2 data. We had to exclude 3 and 9 participants for Day 1 and Day 2 analyses, respectively, because they had less than 7 trials in at least one of the

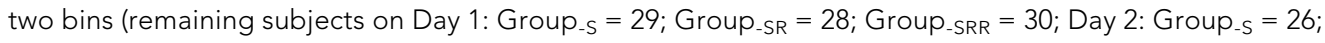
Group $_{-S R}=27$; Group-SRR $=28$ ). For the remaining participants, an average of $56 \pm 3$ and $39 \pm 2$ trials were included for each bin for Day 1 and Day 2 analyses, respectively. Reinforcement-based changes in Error BTC were compared between the groups through one-way ANOVAs with the factor GroupTYPE.

As a second step, we evaluated how participants adjusted movements when they could only rely on the sensory feedback. We compared Error ${ }_{\mathrm{BTC}}$ following bins of Failure trials of different Error magnitudes

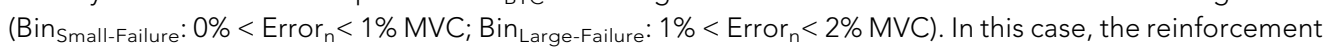
feedback was the same in the two bins and the only difference between the trials consisted in the magnitude of the Error experienced at trial $\left.\right|_{n}$. Again, we first performed a two-way ANOVA with the factors Outcome (Small or Large Failure) and Group TYPE. We then computed sensory-based adjustments as the percentage change in Error BTC $_{\text {in Bin }}$ Large-Failure compared to Bin Small-Failure. This index allowed us to determine how participants adjusted their behavior based on the previous somatosensory experience, in the absence of any difference in the reinforcement feedback obtained.

$$
\text { Sensory }- \text { based adjustments in Error } \text { BTC }=100 \times \frac{\operatorname{ErrorBTC}(\text { BinLarge }- \text { Failure })}{\operatorname{ErrorBTC}(\text { BinSmall }- \text { Failure })}
$$

This analysis was first run on the Day 1 data. We had to exclude 12 participants because they had less than 7 trials in at least one of the two bins (remaining subjects: Group_s $=27$; Group_sR $=28$; Group_sRR $=24$ ). For Day 2, applying the same procedure led to the exclusion of 29 subjects with a lower number of participants in the Group_SRR (15 subjects). For this reason, we ran another analysis where we exceptionally excluded participants only if they had less than 5 trials in one bin. This allowed us to keep a reasonable number of participants in each group (19 subjects excluded; remaining subjects: Group $-\mathrm{S}=24$; Group $_{-\mathrm{SR}}=26$; Group_SRR $=21$ ). Notably, both analyses (i.e., with 7-trials or 5-trials cutoff) gave similar results and we only present the latter in the Results section. For the remaining participants, an average of $47 \pm 3$ and $29 \pm 2$ trials were included for each bin for Day 1 and Day 2 analyses, respectively. Sensory-based changes in Error $_{\text {BTC }}$ were compared between the groups through a one-way ANOVA with the factor Group TYPE.

As a last step, we asked whether the effect of reward on between-trial adjustments in motor commands concerned all aspects of force control, or only specific motor components. To do so, we investigated reinforcement-based and sensory-based adjustments in Force Initiation, $_{\text {Force }}$ AmplError $_{\text {and Force }}$ Variability, using the same method described above for the average Error. We first performed two-way ANOVAs with the 
factors Outcome (reinforcement-based analysis: Success or Failure sensory-based analysis: Small or Large Failure) and Group TYPE. Then, to assess reinforcement-based adjustments, we contrasted between-trial changes in Force Initiation $_{\text {(Force }}$ Initiation-BTC), Force AmplError $_{\text {(Force }}$ AmplError-BTC) and in Force $V_{\text {Variability }}$ (Force Variability-BTC) following Bin $_{\text {Success }}$ and Bin Failure. Sensory-based adjustments were computed by contrasting Force Initiation-BTC, Force $_{\text {AmplError-BTC }}$ and Force Variability-BTC $_{\text {following Bin }}$ Small-Failure $_{\text {for }}$


GrouptYPE.

\section{Group features, muscle fatigue and monetary gains}

As a control, we verified that our 3 groups were comparable in terms of age, success threshold, Target Force, $_{\text {, }}$ and Sensitivity to Reward and to Punishment (i.e., as assessed by the SPSRQ questionnaire). As displayed in Table 1, one-way ANOVAs on these data did not reveal any significant differences between the groups.

We also assessed muscle fatigue on Day 1 and Day 2 (Derosière et al., 2014; Derosiere and Perrey, 2012) by


( $M V C_{\text {PRE }}$ ). The relative change of MVC was not different according to the Group TYPE (Day $1, F_{(2,87)}=$ $0.51, p=0.60 ;$ Day 2, $F_{(2,87)}=0.60, p=0.55 ;$ Table 1$)$. As an additional safety check, we wanted to make sure that the decrements in MVC caused by the training period of Day 1 could not impair performance. To test this, we compared $M V C_{\text {POST }}$ (expressed in \% of $M V C_{\text {PRE }}$ ) with a fixed value of $10 \%$ of $M V C_{\text {PRE }}$ (i.e., corresponding to the Target Force $_{\text {) }}$ through Bonferroni-corrected single sample t-tests. This analysis revealed that $\mathrm{MVC}_{\text {POST }}$ levels were always significantly above the Target Force $_{\text {(Group_s: }} \mathrm{t}_{(29)}=35.84, \mathrm{p}<0.001$; Group_sR: $\mathrm{t}_{(29)}=34.14, \mathrm{p}<0.001$ and Group_SRR: $\left.\mathrm{t}_{(29)}=34.44, \mathrm{p}<0.001\right)$. Hence, force decrements caused by the training were comparable between groups and are unlikely to have limited task performance.

In a final step, we checked that the monetary gains obtained at the end of the experiment were similar between groups. Subjects received a fixed show-up fee corresponding to 10 euros/hour of experiment. In addition, participants also gained a monetary bonus. This bonus was set at 10 euros for subjects in Group_sR and Group_s while it was variable from 0 to 20 euros according to the Group_sRR performance (gain of 8 cents per successful trial in Block_sRR). Importantly, this bonus procedure in Block_sRR was determined to match that obtained in the other groups; it corresponded to $10.4 \pm 0.67$ euros. A t-test revealed that the total ending remuneration was similar across the different Group TYPES $\left(t_{(29)}=0.57 ; p=0.57\right)$. 